\title{
Does cognitive control ability mediate the relationship between reward-related mechanisms, impulsivity, and maladaptive outcomes in adolescence and young adulthood?
}

\author{
Montana McKewen ${ }^{1} \cdot$ Patrick Skippen $^{1} \cdot$ Patrick S. Cooper ${ }^{1,2} \cdot$ Aaron S. W. Wong $^{1,2} \cdot$ Patricia T. Michie $^{1}$. \\ Rhoshel Lenroot $^{3}$ - Frini Karayanidis ${ }^{1,2}$
}

Published online: 22 May 2019

(C) The Psychonomic Society, Inc. 2019

\begin{abstract}
Neurobiological models explain increased risk-taking behaviours in adolescence and young adulthood as arising from staggered development of subcortical reward networks and prefrontal control networks. In this study, we examined whether individual variability in impulsivity and reward-related mechanisms is associated with higher level of engagement in risky behaviours and vulnerability to maladaptive outcomes and whether this relationship is mediated by cognitive control ability. A community sample of adolescents, young adults, and adults (age $=15-35$ years) completed self-report measures and behavioural tasks of cognitive control, impulsivity, and reward-related mechanisms, and self-reported level of maladaptive outcomes. Behavioural, event-related potential (ERP), and multivariate pattern analysis (MVPA) measures of proactive control were derived from a taskswitching paradigm. Adolescents, but not young adults, reported higher levels of impulsivity, reward-seeking behaviours and maladaptive outcomes than adults. They also had lower cognitive control ability, as measured by both self-report and task-based measures. Consistent with models of risk-taking behaviour, self-reported level of cognitive control mediated the relationship between self-reported levels of impulsivity and psychological distress, but the effect was not moderated by age. In contrast, there was no mediation effect of behavioural or EEG-based measures of cognitive control. These findings suggest that individual variability in cognitive control is more crucial to the relationship between risk-taking/impulsivity and outcomes than age itself. They also highlight large differences in measurement between self-report and task-based measures of cognitive control and decision-making under reward conditions, which should be considered in any studies of cognitive control.
\end{abstract}

Keywords Cognitive control $\cdot$ Task-switching $\cdot$ EEG $\cdot$ Risk-taking $\cdot$ Impulsivity $\cdot$ Maladaptive behaviours

Risk-taking during late adolescence and young adulthood forms an integral and adaptive part of the psychological and physical transitions towards adult patterns of behaviour.

Montana McKewen and Patrick Skippen contributed equally to this work.

Frini Karayanidis

frini.karayanidis@newcastle.edu.au

1 Functional Neuroimaging Laboratory, School of Psychology, University of Newcastle, University Drive, Callaghan, NSW 2308, Australia

2 Priority Research Centre for Stroke and Brain Injury, University of Newcastle, Callaghan, Australia

3 Department of Psychiatry and Behavioral Sciences, School of Medicine, University of New Mexico, Albuquerque, NM, USA
Although not all risk-taking is maladaptive (Gullo \& Dawe, 2008; Romer et al., 2017), high-risk behaviours can have adverse long-term consequences (Reyna \& Farley, 2006; Steinberg, 2009) by supporting lifelong patterns of maladaptive behaviours (e.g., addiction, criminality; Moffitt et al., 2011) both in healthy young people and even more so in people with neurodevelopmental disorders (Catts et al., 2013; Steinberg, 2009). Recent models of risk-taking in young people (e.g., Casey, 2015; Steinberg, 2007) posit that heightened risk-taking behaviour and associated maladaptive outcomes during adolescence and young adulthood arise from differences in the rate of maturation of brain networks. Frontal cognitive control networks that are involved in guiding goal-directed behaviour do not mature until well into adulthood, whereas cortico-subcortical networks that support reward-related mechanisms mature during adolescence. This 
is thought to result in heightened sensitivity to the potential rewards that may be associated with engaging in risky behaviours, which, in combination with less efficient control networks, results in poor decision-making, especially under high incentive conditions (Shulman et al., 2016).

Consistent with these models, core cognitive control processes (i.e., working memory, set-shifting, common executive function (EF); Miyake \& Friedman, 2012) continue to develop throughout adolescence and into young adulthood (Karayanidis, Jamadar \& Sanday, 2013; Luna, Garver, Urban, Lazar \& Sweeney, 2004; Ridderinkhof, Band \& Logan, 1999). These processes rely on broadly distributed frontal networks (Gratton, Sun \& Peterson, 2018) that also show protracted development in both structure (Paus, 2005; Gogtay et al., 2004) and connectivity with cortical and subcortical regions (Hwang, Velanova \& Luna, 2010; Stevens, Kiehl, Pearlson \& Calhoun, 2007). Functional differences also have been shown. For instance, compared with adults, adolescents show less engagement of frontoparietal control networks during risky decision making (Eshel, Nelson, Blair, Pine \& Ernst, 2007; Fecteau et al., 2007) and greater activation of reward-related networks when processing appetitive cues (Sommerville, Hare \& Casey, 2011, van Leijenhorst et al., 2009).

There also is substantial evidence linking cognitive control and risk-taking (Peeters, Oldehinkel \& Vollebergh, 2017; Magar, Phillips \& Hosie, 2008), impulsivity (Stahl et al., 2014), and maladaptive outcomes in later life (Moffitt et al., 2011). Likewise, high reward seeking and impulsivity, measured both behaviourally and through self-report, are associated with greater engagement in maladaptive and risk-taking behaviours (Castellanos-Ryan et al., 2013; Derefinko et al., 2014; Donohew et al., 2000; Mackillip et al., 2016; Stahl et al., 2014). However, the relationship between the developmental trajectories of brain networks associated with reward mechanisms and cognitive control is complex, showing dynamic interactions extending well beyond adolescence (Pfeifer \& Allen, 2012; Romer, Reyna \& Satterwaite, 2017). For instance, level of cognitive control (van Leijenhorst et al., 2010) and sensitivity to feedback (Koolschijn, Schel, de Rooij, Rombouts \& Crone, 2011) were found to be stronger predictors of brain maturation than age alone, consistent with substantial individual variability in the rate of development of these processes (Romer, Reyna \& Satterwaite, 2017).

Moreover, there are still large gaps in our understanding of how cognitive control and reward mechanisms relate to intraand inter-individual variability in impulsivity and risk-taking behaviours. For instance, within dual-systems models, impulsivity is sometimes encompassed under psychosocial maturity, a composite construct that also includes risk perception, sensation seeking (Steinberg, 2008), and other times used as a proxy for response inhibition, a core cognitive control process (Steinberg, 2010). These distinct definitions are consistent with Dawe's conceptualisation of impulsivity as a multifaceted construct that includes two key domains: one relating to reward sensitivity or drive and the other to disinhibition (Dawe \& Loxton, 2004; Gullo \& Dawe, 2008). Few studies have concurrently examined individual variability in the relationship between cognitive control, impulsivity, reward-related mechanisms, and outcome behaviours in adolescence and young adulthood (Bjork \& Pardini, 2015; Casey et al., 2011; Laurens et al., 2007; Pfeifer \& Allen, 2012; Steinberg, 2007; Shulman et al., 2016).

In this study, we used mediation analyses to test the implicit assumption of risk-taking models of adolescence and young adulthood that cognitive control ability mediates the effect of impulsivity and reward-seeking on level of engagement in risky behaviours and maladaptive outcomes (Casey et al., 2010; Steinberg, 2009). We hypothesised that higher levels of impulsivity and reward-seeking are associated with greater engagement in risky behaviours and maladaptive outcomes and that this relationship is mediated by level of cognitive control. Adolescents are expected to exhibit higher impulsivity and reward-seeking, as well as lower cognitive control than adults. Because variability in cognitive control is expected to be greater in adolescence, we hypothesised that the mediating effect of cognitive control on the relationship between impulsivity/reward-seeking and outcome behaviours will be greater in this group.

Reward-related mechanisms cover a broad range of constructs that are defined and operationalised differently across the literature (Harden et al., 2017; Duell et al., 2016; Nigg, 2017). For example, risk-taking is impulsive when the behaviour is engaged without thought of consequence, yet also can be quite planned and thoughtout (Nigg, 2017). The association between impulsivity, reward mechanisms, cognitive control ability, and maladaptive outcomes has been shown to be weak and to vary with the level of measurement (Skippen et al., 2019; for review see Stahl et al., 2014) as well as different researchers' definition of such terms (Nigg, 2017; Romer et al., 2017). We included both self-report and behavioural measures of impulsivity and reward-seeking to capture both levels of measurement and identify independent patterns. Furthermore, to target real-world behaviours, outcome measures focussed on self-reported level of engagement in risky behaviours, quality of life, and psychological well-being.

Most imaging studies of risk-taking behaviour have used magnetic resonance imaging (MRI) measures to identify variability across brain regions and networks involved in cognitive control and reward processes. However, electroencephalography (EEG) methods can better capture the temporal variability of complex, dynamic processes associated with cognitive control processes (Gratton, Cooper, Fabiani, Carter \& Karayanidis, 2018) and may be more sensitive to capturing developmental changes in cognition across the lifespan (Segalowitz, Santesso, \& Jetha, 2010; Uhlhaas, Roux, Rodriguez, Rotarska-Jagiela, \& Singer, 2010). 
Like the constructs of impulsivity and reward seeking, cognitive control also can be defined a number of different ways (Harden et al., 2017). In this study, we describe cognitive control as the higher-order executive functions, planning, working memory, task-set updating (Miyake \& Friedman, 2012). We obtained measurements of cognitive control at different levels (EEG, behaviour, self-report) to determine whether cognitive control processes differ in their mediation of the relationship between reward-seeking and maladaptive outcomes. We used the cued-trials task-switching paradigm (Karayanidis et al., 2010; see Jamadar, Thienel \& Karayanidis, 2015 for task-switching paradigms and associated cognitive processes) that produces behavioural and eventrelated potential (ERP) measures sensitive to proactive cognitive control processes (Braver, 2012). These processes are involved in anticipating and preparing for a change in contextual demands to optimise performance and have been shown to mature more slowly in childhood and adolescence (Karayanidis et al., 2013; Munakata et al., 2012). We sought to optimise the opportunity to identify specific proactive control processes that impact risk-taking behaviour and quality of life by deriving multiple sensitive measures of proactive control. Behavioural switch cost and mixing cost measures target set-shifting ability and working memory load, respectivelytwo core cognitive control processes (Miyake and Friedman, 2012) that have been found to engage frontoparietal (Cooper et al., 2016; Mansfield et al., 2012) and frontostriatal (Mansfield et al., 2011) control networks. We extracted measures of ERP components during the cue-target interval (CTI) to examine individual variability in the engagement of proactive control processes involved in preparing to shift between tasks-sets. In addition, we implemented multivariate pattern analysis (MVPA) of the EEG data (Bode et al., 2018) to characterise individual variability in the timing and accuracy of working memory and set-shifting processes involved in mixing cost and switch cost, respectively. We argue that the accuracy of discrimination of EEG epochs from trials that differ in set-shifting demands (or working memory demands) is indicative of processing efficiency and that greater discrimination is suggestive of more efficient implementation of cognitive control.

\section{Methods}

\section{Participants}

A community sample was recruited from schools, businesses, community organisations, and tertiary education centres in the Central Coast and Hunter regions of New South Wales, Australia. Participants who reported having received a clinical diagnosis of psychological or neurological conditions were excluded. Of the 238 participants who were included in the
Age-ility project (Karayanidis et al. 2016; Cooper et al., 2015), 215 participants completed the EEG session (Figure 1). Twenty-two percent were removed because of task-switching data problems $(\mathrm{n}=4)$ or missing data $(\mathrm{n}=$ 43), leaving a final sample of 168 participants $(20.91 \pm 4.75$ yr, range $15-35 \mathrm{yr}, 91 \%$ right handed). Men and women $(56.5 \%)$ did not differ in age $(21.5 \pm 5.0 \mathrm{yr}, 20.2 \pm 4.4 \mathrm{yr}$, respectively $F_{(1,166)}=3.346, p=0.069$ ). This sample did not differ from the 70 participants who had no EEG or incomplete data on any common variables, including age, sex, and neuropsychological measures (all $p>0.162$ ).

The protocol was approved by the University of Newcastle Human Research Ethics Committee (HREC: H-2012-0157). Participants (and their parents/guardians, if aged under $18 \mathrm{yr}$ ) gave written, informed consent and were reimbursed $\$ 20 / \mathrm{hr}$.

We examined scatterplots of age against other variables (see below) and fitted a Loess curve (see online supplementary materials; https://osf.io/hs9me/), which indicated that the effect of age was not strongly linear, consistent with literature, suggesting continuing development of cognitive mechanisms from adolescence into young adulthood reaching a plateau thereafter. Consequently, we defined three age groups: Adolescent (15-18 yr, $\mathrm{n}=62,33$ females, $16.8 \pm$ $1.2 \mathrm{yr}$ ), Young Adult (19-24 yr, $\mathrm{n}=74,39$ females, $20.9 \pm 1.6$ yr), and Adult (25-35 yr, $\mathrm{n}=32,23$ females, $28.9 \pm 3.7)$. Sex did not differ across groups $(p>0.15)$.

We analysed all variables using a three age-group (Adolescent, Young Adult, Adult) $\times$ two Sex ANOVA, with repeated measures factors specified where appropriate (see in each section of Results). Significant age-group effects were followed by simple contrasts comparing Adolescents vs. Young Adults, Adolescents vs. Adults, and Young Adults vs. Adults with Greenhouse-Geisser corrections for the assumption of sphericity violations. We report significant results with exact $p$ values, except where $<0.0001$, and include full list of nonsignificant results at https://osf.io/hs $9 \mathrm{me} /$.

\section{Procedure}

Participants completed three testing sessions (Karayanidis et al. 2016). The first session included a neuropsychological battery focussing on cognitive control and decision-making, as well as practice on the task-switching paradigm. Participants were given a series of questionnaires, including measures of cognitive control, impulsivity, reward-seeking, risk behaviours, and psychological well-being, to complete in the laboratory or at home to be returned for the second session. The second session occurred 2 weeks later and included further task practice and performance of the taskswitching paradigm while EEG was recorded. The third session included an MRI scan; the results of which are not reported here (Karayanidis et al. 2016). 


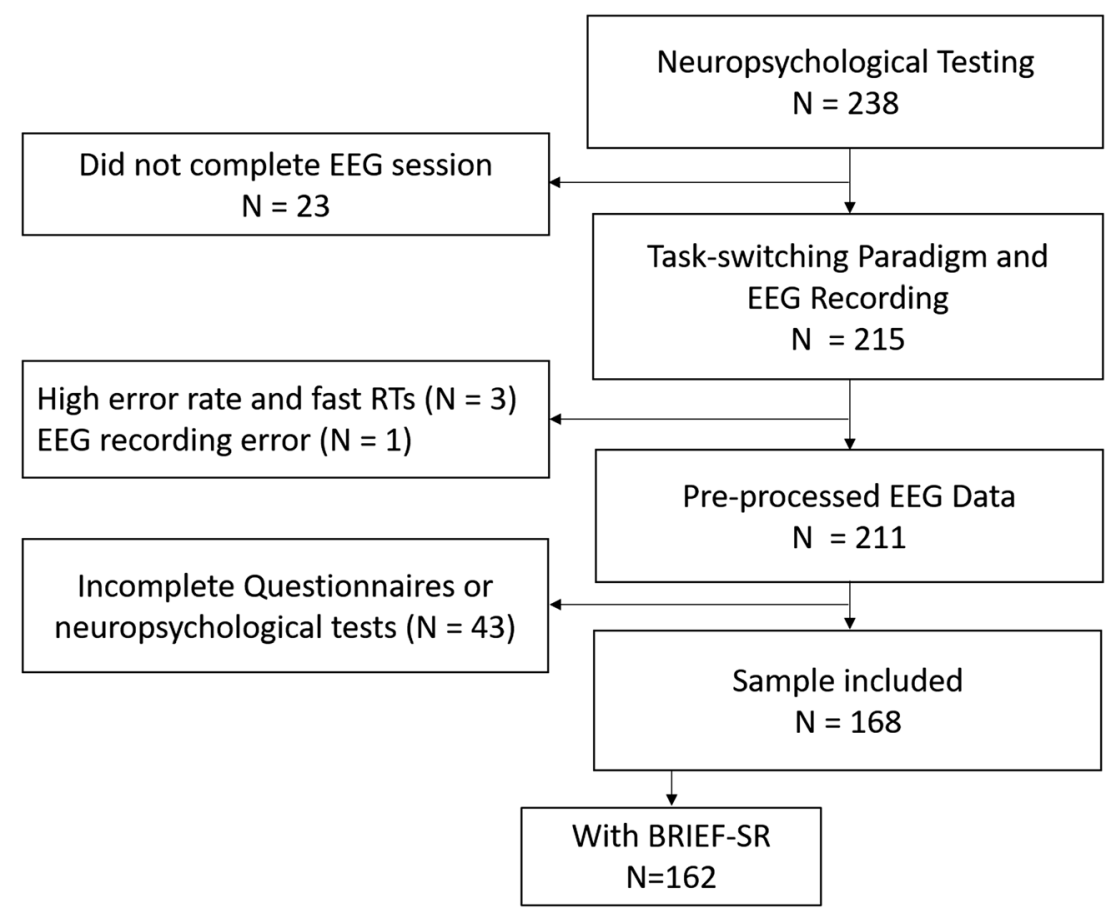

Fig. 1. Participant attrition flowchart. Note: BRIEF-SR = Behavioural Rating Inventory of Executive Function Self-Report

Table 1 lists all tasks and questionnaires and the measures analysed from each. Predictor variables included measures that characterise the individual in terms of constructs, such as impulsivity, sensation seeking, risk appraisal, and risky decision-making. Outcome variables included self-report measures of engagement in risky behaviours, psychological distress, and quality of life. Cognitive control variables were derived from well-established tasks of reasoning, problem solving, and working memory, the cued-trials task-switching paradigm (Karayanidis et al., 2016), as well as the self-report version of the Behavioural Rating Inventory of Executive Function (BRIEF).

Missing data Five participants had minimal missing data, and we used the following approaches to avoid excluding the entire case. Two participants had more than two items missing on the SSS and the Barratt Impulsivity Scale-11 (BIS-11), respectively. We used regression imputation to estimate missing values (Saunders et al., 2006). For each participant, we ran regression across all participants on variables related to the scale with missing data. The resulting linear equation $(y=m x+b)$ was solved for the missing data point (y). Missing data for each participant was imputed by averaging all estimates (y values).

Another participant's missing K10 total score was substituted with the mean of the entire sample. On the Sensation Seeking Scale (SSS), one participant had one missing response, and another had two missing responses. These missing values were replaced with the individual's mean score on the corresponding subscale, and the total scores was computed using the replaced values.

\section{Variable Reduction}

As many of the variables derived from self-report and behavioural measures tap into partially overlapping constructs, we used principal components analysis (PCA) to derive separate PCA models for variables characterised as predictors $(n=17)$, outcomes $(n=20)$, and cognitive control measures $(n=10)$ from the neuropsychological tasks (Table 1$){ }^{1}$

PCA relies on the variable correlation matrix to identify components that maximise the total variance within these measures. We assessed all measures for outliers or other biases that may affect the linearity of correlations but did not need to remove any variables due to outlying values. To correct for nonlinear biases, we took the natural log of the CARE Past Frequency and WHOQol scores. For ease of interpretation, some variables were reversed so that for all PCA component loadings high scores indicate higher level of risk-taking/impulsivity (predictor components), poorer life outcomes (i.e., higher psychological distress, more risky behaviours, poorer quality of life, outcome components), and poorer cognitive control ability (cognitive control components).

In a PCA model, each component is a linear combination of all variables and will fit as much of the variability as possible into the first component. The remaining variance is attributed to the subsequent component, and so on. The original variables are weighted by their contribution to explaining the

\footnotetext{
${ }^{1}$ The BRIEF scale was omitted from the cognitive control PCA, because we had to use T-scores to compare scores from Child ( $<18 \mathrm{yr})$ and Adult $(>18 \mathrm{yr})$ versions of the scale. Note that only 162 of the 168 participants completed the BRIEF.
} 
Table 1 List of (A) Predictor variables used to measure impulsivity and risk propensity, (B) Outcome variables used to assess risk behaviours and wellbeing, and (C) Cognitive control measures

\section{A. PREDICTOR VARIABLES}

Barratt Impulsivity Scale (BIS-11; Patton, Stanford \& Barratt, 1995) A 30-item, self-report questionnaire that measures perceived level of impulsive behaviours and preferences. We used the three $2^{\text {nd }}$ order factors: attentional, motor, and non-planning impulsivity.

Cognitive Appraisal of Risky Events - 2. Perceived Risk Benefit (CARE-Perceived Risk Benefit; Fromme, Katz \& Rivet, 1997)

Sensation Seeking Scale (SSS; Zuckerman, Kolin, Price \& Zoob, 1964)

Information Sampling Task (IST; Cambridge Cognition, 2006)

Cambridge Gambling Task (CGT; Cambridge Cognition, 2006)

\section{B. OUTCOME MEASURES}

Cognitive Appraisal of Risky Events - 1. Past Event Frequency (CARE-Past Frequency; Fromme, Katz \& Rivet, 1997)

Depression Anxiety Stress Scale (DASS; Antony, Bieling, Cox, Enns \& Swinson, 1998)

Difficulty in Emotional Regulation Scale (DERS; Gratz \& Roemer, 2004)

World Health Organisation Quality of Life (WHOQoL; WHOQOL Group, 1998)

Kessler Psychological Distress Scale (K-10; Kessler et al., 2002)

Suicidal Behaviour Questionnaire (SBQ; Osman et al., 2001)

\section{COGNITIVE CONTROL MEASURES}

Matrix Reasoning, Wechsler Abbreviated Scale of Intelligence-II (WASI-II; Wechsler, 2011)
A 30-item, self-report questionnaire that measures perceived benefit of engaging in risky behaviours including Drug use, Aggression/Illegal activity, Risky Sexual Behaviour, Hazardous Drinking, and missing Work/study commitments.

A 40-item, self-report questionnaire that assesses perceived preference for a range of activities across four subscales: Thrill and Adventure Seeking, Disinhibition, Experience Seeking, Boredom Susceptibility.

IST provides behavioural measures of reflection impulsivity, the tendency to evaluate information before making a decision. Each trial includes a $5 \times 5$ array of grey tiles that are yellow or blue on the reverse surface. Participants are asked to decide whether the array is hiding more yellow or blue tiles, and receive points reward for correct decisions. In different conditions, the reward value decreases (decreasing win) or increases (ascending win) with every tile opened. $P$ (Correct) is the probability of being correct that the participant tolerates at the point of decision-making. We report data only from the descending win conditions, as there was no variability in the ascending win condition.

CGT measures risk-taking behaviour and delay aversion. Participants are presented with ten boxes that differ in red:blue ratio $(9: 1$ to $6: 4)$ and are asked to respond which colour is most likely to contain a hidden token. On each trial, participants can delay their response to increase or reduce the bet value associated with that choice. In different blocks, the bet value increases or decreases over the course of the trial. Delay Aversion measures the inability to wait to optimise reward (the difference in bet value between ascending and descending bet conditions, when controlling for risk level, high score $=$ greater aversion to delaying response). We used ratios 6:4 and 9:1, which showed the largest individual differences. Risk Adjustment measures how well one can adjust their risk taking behaviour to maximise reward as the extent to which the behaviour is moderated by the ratio of red to blue boxes, when controlling for delay.

A 30-item, self-report questionnaire that measures the frequency of engaging in risky behaviours including Drug use, Aggression/Illegal activity, Risky Sexual Behaviour, Hazardous Drinking, and missing Work/study commitments, in the past six months.

A 21-item, self-report questionnaire with three scales that measure perceived level of depressive, anxiety and stress symptoms.

A 36-item, self-report questionnaire that assesses perceived difficulty in regulating one's emotions across six subscales: Non-acceptance of emotional responses, Difficulty engaging in goal directed behaviour, Impulse control difficulties, Lack of emotional awareness, Limited access to emotion regulation strategies, and Lack of emotional clarity.

A 26-item, self-report questionnaire that assesses perceived quality of life across four domains: Physical, Psychological, Social, and Environmental.

A 10-item, self-report questionnaire of perceived level of psychological distress.

A 4-item, self-report questionnaire of suicidal thoughts and behaviour that asks about lifetime ideation, ideation frequency, threat of attempt, and future likelihood of suicidal behaviour.

A task of fluid intelligence where participants select the logically following item in a pattern or the missing piece in a matrix. We report the total raw score. 
Table 1 (continued)

Trail-Making Test (TMT; Reitan, 1959)

Verbal Fluency (FAS Verbal Fluency task; Lezak, Howieson, Loring \& Fischer, 2004)

Digit Span, Wechsler Adult Intelligence Scale-IV

(WAIS-IV; Wechsler, 2008)

Spatial Working Memory (SWM; Cambridge Cognition, 2006)

Spatial Span (SSP; Cambridge Cognition, 2006)

Stockings of Cambridge (SOC; Cambridge Cognition, 2006)

Behavioural Rating Index of Executive Functions (BRIEF; Roth, Isquith \& Gioia, 2005) ${ }^{\mathrm{a}}$
A task of set-shifting ability. It measures the time taken to trace a line to link numbers from 1-25 presented in a random configuration (Part A) and alternate between letters and number in sequence (Part B). The B-A score indicates set-shifting ability when controlling for perceptual and motor speed. A task of flexible thinking. Measures the number of unique words produced in 1 min starting with the letters $\mathrm{F}, \mathrm{A}$, and $\mathrm{S}$, in different blocks.

Verbal fluency total score is the total number of correct words summed across all three blocks.

A task of short-term (forward) and working memory (backward, sequencing) ability. Span length is number of digits correctly recited. We report Span Length using forward-backward and forward-sequencing to measure working memory when controlling for short-term memory.

A task of the ability to retain and manipulation of visuospatial information. A token is hidden under a different box in a random array of boxes on each trial. Participant seek the token without opening the same box twice.

Total Error score measures working memory (i.e., the number of times the same box is checked). We report Strategy which measures strategy formation (i.e., development of a consistent approach to solving consecutive problems).

A task of visuospatial working memory capacity. An array of white boxes is displayed. On each trial, a sequence of boxes briefly change colour one at a time in a variable sequence, and then participants are asked to tap the boxes that changed in the correct sequence. We report Span length, the longest sequence correctly recalled.

A task of spatial planning, spatial working memory and motor control. Participants solve problems by rearranging coloured balls in one display to match that of the test display. Problems can be solved in a minimum of 2-5 moves. We report Mean Moves, the mean number of moves to solve a problem for 4 and 5 move problems.

An 86-item, self-report questionnaire of executive function which derives two main indices. The Behavioural Regulation Index (BRI) measures perceived ability to control behaviour and emotional responses. The Metacognition Index (MI) measures perceived ability on higher order processes including planning, organisation, and maintaining attention. A higher score on these indices indicates higher levels of executive dysfunction. Note: For BRIEF, $N=162$.

See Methods for task details.

Task-switching paradigm ${ }^{\mathrm{a}}$ (Karayanidis et al., 2009)

Total score is used unless stated otherwise

${ }^{a}$ These measures were not included in the PCA - see Footnote 1 for explanation.

variance in each linear dimension, making the interpretation of components difficult in some cases (Jolliffe, 2011). Consequently, we used oblique component rotation, which unlike traditional orthogonal (e.g., Varimax) rotation allows the rotated components to correlate. This results in a more realistic component structure (given that the variables entered into each PCA model intercorrelate across the hypothesised component structure; e.g., measures of psychological distress (e.g., DASS) intercorrelated with measures of quality of life (WHOQol), and self-report risk behaviours (CARE Past Frequency).

The PCA was conducted using the "Psych" package (Revelle, 2018) in the R statistical modelling program ( $\mathrm{R}$ Core Team, 2017). Z-scored data were entered into the fa.parallel function to determine the number of components to extract, using Horn's (1965) parallel method of determining the number of factors. This function displays a standard scree plot of the eigenvalues, as well as a scree of a random data matrix of the same size, and components whose eigenvalues are larger than the random sample are retained. The analysis suggested five components for the predictor: three for the outcome, and two for the cognitive control variables. These were extracted using the principal function with default settings. The "GPArotation" package (Bernaards \& Jennrich, 2005) was used to conduct the oblique rotation ("Oblimin"), and we examined the loadings of each rotated component to interpret the components.

Because some models contained a small subject:variable ratio (e.g., outcomes $=186: 20$ ), we obtained a number of descriptive statistics about each model to assist with the interpretation of the resulting model structure. The variable loading strength and the number of variables loading on each component were relatively high, indicating low error and good fit (Osborne \& Costello, 2004). The communality of each 
variable, which particularly in exploratory factor analysis is considered a measure of the reliability of the variable (Gaston, 2008), as well as the confidence intervals of loadings and complexity, is available in the online supplementary materials (https://osf.io/hs9me/). Table 2 shows the loadings of each variable on the rotated components for predictor, outcome, and neuropsychological variables.

The five rotated Predictor components accounted for $64 \%$ of variance: (1) Risk Propensity contained strong contributions from measures that ask about the desire to engage in (SSS) and the perceived benefit of (CARE-2) risky behaviours; (2) Impulsivity contained strongest contribution from the BIS-11 subscales; (3) Delay Aversion included selfreported SSS Boredom Susceptibility and behavioural measures of delay aversion from the CGT; (4) Impulsive Decisions consisted of behavioural measures of risk adjustment from the CGT, as well as the Probability Correct from the IST; and (5) Work \& Aggression contained strongest contributions from CARE-2 scales of perceived benefit of aggression and lax work practices.

The PCA model from the outcome variables produced a three rotated component structure that explained $56 \%$ of variance: (1) Psychological Distress contained the DASS, K-10, and SBQ variables, and most of the DERS scales; (2) Quality of Life contained the WHOQoL scales and DERS Awareness scale; and (3) Risky Behaviours included the CARE PastFrequency scales.

Only two components were returned from the PCA model on the neuropsychological measures of cognitive control and accounted for $44 \%$ of the variance: (1) General-Executive Function (General-EF) contained the highest loadings from Trail-Making Task, Matrix Reasoning, Verbal Fluency, both SWM scores, as well as SOC; and (2) Working Memory Span contained strong loadings from the Digit Span measures, with weaker cross loading from SSP.

\section{Task-switching paradigm}

We used the cued-trials task switching paradigm with three tasks developed by Karayanidis et al. (2009).

Stimuli and task parameters A grey circle ( $5^{\circ}$ visual angle) was continuously displayed and divided into six sections, with adjacent sections mapped to one of three tasks (Figure 2A). The target was a pair of characters (e.g., grey A4) with three dimensions: one relevant to the task (e.g., if the target was in a letter section, "A" would be mapped to a left response), one irrelevant dimension that was always incongruently mapped to the relevant task's response (e.g., "4" mapped to a right response) and a neutral dimension that was not mapped to any response (e.g., grey). A cue indicating the relevant task for this trial preceded the target by $1,000 \mathrm{~ms}$ (i.e., a highlight over two adjacent regions of the circle; Figure 2B). In single- task blocks, the cue remained in the same position throughout the block, indicating that the same task was to be repeated (allrepeat trials). In mixed-task blocks, on repeat trials $(25 \%$; Figure 2Ci), the cue remained in the same position on consecutive trials. Repeat trials were identical to all-repeat trials but were interspersed with switch trials (25\%; Figure 2Cii) on which the cue changed position and highlighted segments associated with one of the other two tasks. The remaining $50 \%$ of trials used partially informative cues and are not used here (see Karayanidis et al., 2009 for more task details). Targets were presented for $5000 \mathrm{~ms}$ or until a response was emitted, and the next cue occurred 400-ms later. Incorrect responses resulted in an error feedback tone. Participants completed two training sessions (1,320 practice trials) before undertaking 10 mixed-task blocks ( 77 trials/block) and three single-task blocks (53 trials/block) while EEG was recorded.

We derived two key task-switching measures. Switch cost is the difference in RT and error rate between switch and repeat trials, and reflects processes involved in task-set updating on switch trials (Karayanidis et al., 2009). Mixing cost is the difference in RT and error rate between repeat and all-repeat trials and reflects increased working memory load on mixed-task compared to single-task blocks (Los, 1996).

EEG Recording and Processing EEG was continuously recorded relative to an amplifier reference voltage using an ActiveTwo Biosemi EEG system $(2048 \mathrm{~Hz}$, bandpass filter of DC-400 Hz) from 64 scalp electrodes, left and right mastoids, bilateral outer canthi, and supra/infraorbital sites. Common mode sense (CMS) and driven right leg (DRL) electrodes were positioned inferior to $\mathrm{P} 1$ and $\mathrm{P} 2$, respectively. EEG was processed in MATLAB through a pipeline utilising Fieldtrip (Oostenveld et al., 2011), CSD Toolbox (Kayser \& Tenke, 2006) and in-house functions (Wong \& Cooper). EEG was re-referenced off-line to electrode $\mathrm{Cz}$ and then downsampled from $2,048 \mathrm{~Hz}$ to $512 \mathrm{~Hz}$ (using the fieldtrip $f t$ preproc_resample function; zero-phase anti-aliasing filter with a low-pass cutoff frequency of $245 \mathrm{~Hz}$ ). Data were high pass and notch filtered to remove line noise and lowfrequency drift (high pass: $0.1 \mathrm{~Hz}$, forward phase; $50 \mathrm{~Hz}$ notch: zero phase). Excessively noisy channels were identified with visual inspection and excluded (average $1.7 \pm 4.1$ channels per participant). Epochs were extracted from $-1,000 \mathrm{~ms}$ to 3,500 ms with respect to cue onset. Blink and vertical eyemovement artefacts were identified and removed by a trained observer using Independent Components Analysis (ICA) with the fastICA algorithm (Hyvärinen \& Oja, 2000; $1.38 \pm 0.76$ components). The remaining components were projected back into sensor (electrode) space. The data were low pass filtered (30 Hz, zero-phase) and trials with residual artefact larger than $\pm 120 \mu \mathrm{V}$ were deleted. On average, pre-processing resulted in $110.92 \pm 23.85$ all-repeat, $131.51 \pm 24.94$ repeat, and 126.34 \pm 26.62 switch trials per participant, with a minimum of 30 
Table 2 Loadings of (A) Predictor, (B) Outcome, and (C) Cognitive Control components derived from the principal component analyses

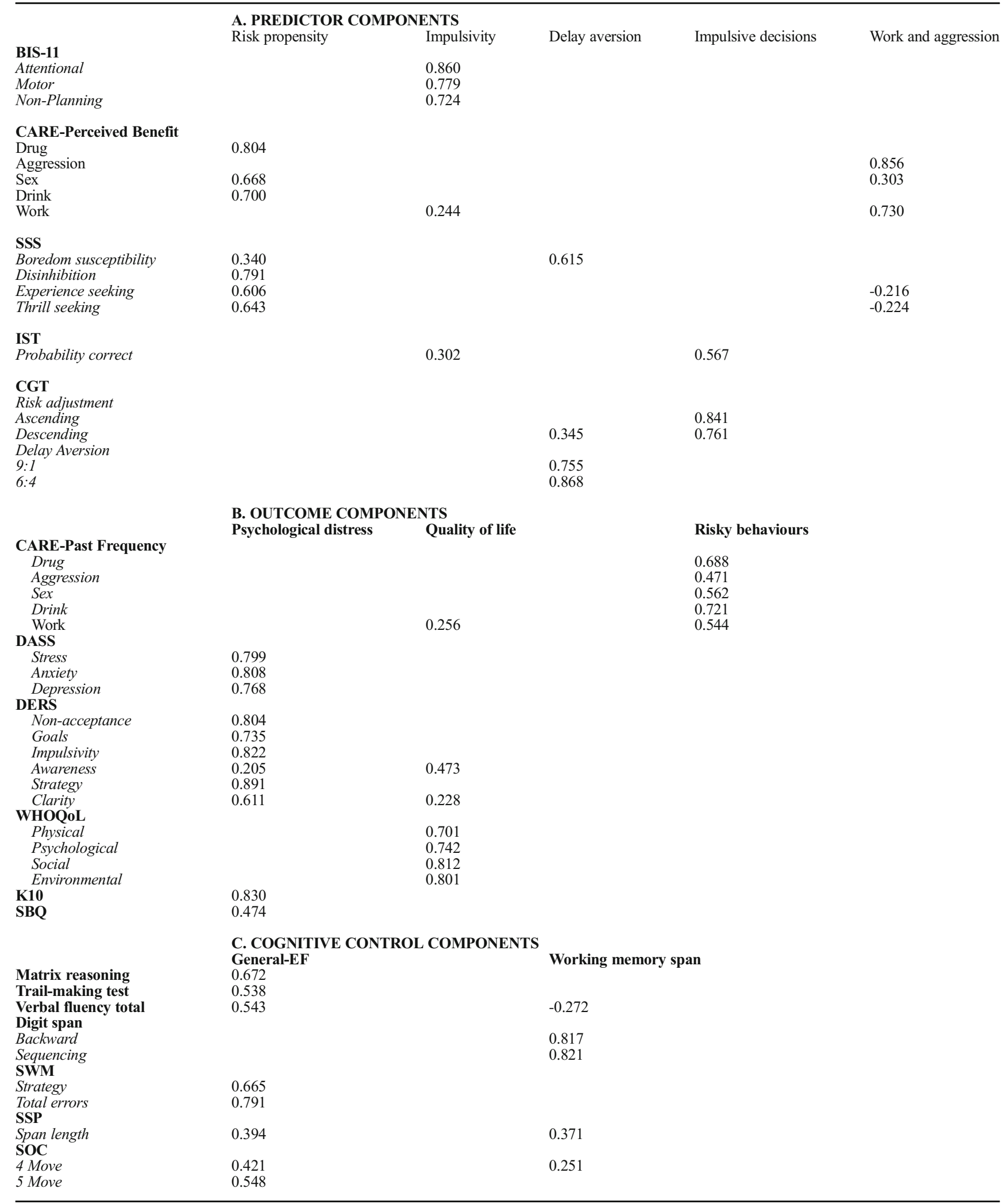

Loadings between -0.2 and 0.2 are omitted for ease of interpretation. Component names are selected to represent the contributing measures.

Full loadings and other descriptive statistics can be found in the supplementary materials. EF = Executive Function. 
trials per trial type per participant. The surface Laplacian transformation was computed and a spherical spline function was applied across all channels, with a spline flexibility parameter, $\mathrm{m}=4$, for increased rigidness (Kayser \& Tenke, 2015). An iterative process was used to solve a Legendre differential equation to obtain the surface Laplacian and surface potential matrices (Kayser \& Tenke, 2006). As the EEG signal is transformed based on the second partial derivate of the signal $(\mu \mathrm{V})$ over a spatial area $\left(\mathrm{cm}^{2}\right.$ - i.e., the scalp), the measurement scale is $\mu \mathrm{V} / \mathrm{cm}^{2}$ (Kayser \& Tenke, 2006, 2015).

ERP Measures Cue-locked ERP average waveforms were derived for each trial type (all-repeat, repeat, switch) from $-200 \mathrm{~ms}$ to $1,200 \mathrm{~ms}$ peri-cue, with a $\pm 50 \mathrm{~ms}$ baseline. Mixing cost (repeat minus all-repeat) and switch cost (switch minus repeat) difference waveforms were extracted. The topography of significant mixing and switch effects across the cue-target interval were inspected in $\approx 10 \mathrm{~ms}$ bins ( 5 sample points at $512 \mathrm{~Hz})$. Paired samples $t$-tests $(\alpha<0.005$, FDR corrected; Benjamini \& Yekutieli, 2001) were conducted to determine "hotspots" (i.e., electrode clusters and time windows) of significant posterior mixing and switch positivities typically obtained from this paradigm (Karayanidis et al., 2009). Table 3 shows early and late positivity hotspots identified for mixing and switch difference waveforms. For each hotspot, we measured peak amplitude over that time window at each electrode using an average measure over four sample points either side of the maximum (i.e., $\approx 18 \mathrm{~ms}$ ), as well as the latency of the peak amplitude. Peak amplitude was compared across electrodes within that hotspot, and the electrode with the largest peak amplitude was used to derive peak amplitude and latency for that hotspot.

Multivariate Pattern Analysis (MVPA) was implemented in the Decision Decoding Toolbox (DDTBOX, Bode et al.,
2018) to classify epochs of spatio-temporal EEG data measured on the two-dimensional surface of the scalp in a binary manner. This technique is based on a support vector machine (SVM; Cortes \& Vapnik, 1995), which uses an optimized linear kernel (LIBLINEAR, Fan et al., 2008) to obtain the hyperplane that maximises the margin of separation between two classes or conditions.

We used MVPA to discriminate between ERPs for switch and repeat trials, corresponding to switch cost, and ERPs for repeat and all-repeat, corresponding to mixing cost. For each discrimination, the time of interest ranged $50-2,050 \mathrm{~ms}$ postcue (peri-cue baseline $\pm 50 \mathrm{~ms}$ ), so as to include a post-target interval. A tenfold cross-validation training process was repeated 10 times for each individual's EEG trials on every spatiotemporal EEG feature, resulting in 100 classification rates per time bin per individual. A total of 320 spatiotemporal EEG features (mean amplitude from nonoverlapping 10-ms sliding windows at 64 channels) were used to train an SVM for each time bin (210 time bins per epoch). For each individual, a null classification rate was calculated using the above method, by randomly permuting condition labels. Across the sample, 6.762 million linear classifiers were trained across all discriminations used in this analysis.

For each individual, we measured the maximum classification rate over the $50-2,050 \mathrm{~ms}$ interval and the latency at which this occurred. The signal was smoothed using a 10-point time domain moving average filter, and peak analysis was used to identify the global maximum classification rate. Specifically, a linear search was used to identify all local maxima in the signal and identify the global maximum. A local maximum was identified as the global maximum, if its value exceeded that of the previous global maximum by a certain threshold. This threshold was defined separately for each discrimination task using an optimising process that favoured the first maximum
A Task structure

\begin{tabular}{|lcc|}
\hline \multicolumn{3}{|c|}{$\begin{array}{l}\text { Color } \\
\end{array}$} \\
\end{tabular}

Fig. 2. Cued-trials task switching paradigm. (A) Display structure indicating mapping of adjacent segments to letter, digit, and colour classification and example of stimulus-response mapping. (B) Single trial example. A cue is presented $400 \mathrm{~ms}$ after the response to the previous trial and highlights two adjacent segments (corresponding here to the letter task), indicating that the next target will appear in one of these segments. After
B Trial N - 1
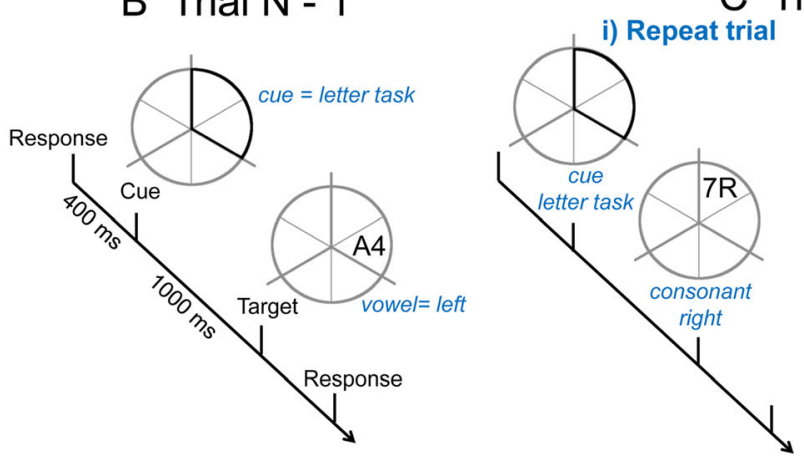

C Trial $\mathrm{N}$

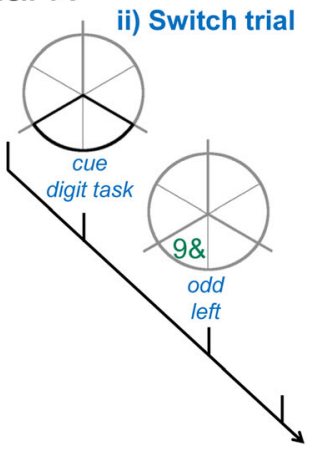

1,000 ms, a target appears in one of the highlighted segments and participants are required to respond to the target. (C) The following trial $(\mathrm{N}-1)$ could be a i) repeat trial, i.e., the same two segments are highlighted and the same task is performed, or ii) switch trial, i.e., the cue highlights segments associated with one of the other two tasks and validly indicates which task to perform on the target 
Table 3 Electrodes and time windows of event related potential (ERP) components representing switch and mixing costs

\begin{tabular}{llll}
\hline Cost Type & ERP label & Electrodes & Time window (ms) \\
\hline Switch & Early-cue positivity & PO7,PO3,POz,PO4,PO8,O1,Oz,O2 & $250-320$ \\
& Late-cue positivity & CPz,CP1,CP3,CP2,CP4,Pz,P1,P3,P2,P4,POz,PO3,PO4 & $340-550$ \\
Mix & Early-cue positivity & P3,P1,Pz,P2,P4,PO3,POz,PO4 & $280-450$ \\
& Late-cue positivity & P1,Pz,P2,PO3,POz,PO4 & $452-900$ \\
\hline
\end{tabular}

classification, unless the subsequent one was substantially greater (i.e., avoid a minor increase in rate producing a disproportionate and potentially artificial increase in latency), hence minimising the number of subjects with a posttarget maximum classification. The threshold was $3.64 \%$ for the switch versus repeat discrimination, and $3.96 \%$ for the repeat versus all-repeat discrimination.

Analyses Warmup trials ( 5 per block), trials with RT $<200 \mathrm{~ms}$ or $>3 \mathrm{SD}$ above the individual's mean RT, and error or posterror trials were removed from analyses (on average, $18.5 \pm$ $8 \%$ of trials) of RT, ERP, and MVPA measures. Behavioural (median RT, error rate) and EEG-based (ERPs, MVPA) measures were analysed using GLM in SPSS v21 with Trial Type (all-repeat, repeat, switch) as a repeated measures factor, and Age Group and Sex as between-group factors (see below). Two planned comparisons were used to assess switch cost and mixing cost, with family-wise error Bonferroni correction $(\alpha<0.05 / 2)$ and Greenhouse-Geisser correction for the violations of the assumption of sphericity.

\section{Mediation Analyses}

Mediation analysis was used to assess whether the relationship between predictor $(\mathrm{X})$ and outcome $(\mathrm{Y})$ variables was mediated by cognitive control variables (M). If the total effect of $X$ on $\mathrm{Y}$ was reduced or removed after including $\mathrm{M}$, this was taken as evidence that the relationship between $\mathrm{X}$ and $\mathrm{Y}$ was partially or fully mediated by $\mathrm{M}$, respectively (Baron \& Kenny 1986; MacKinnon 2008). To compare the strength of mediation, we standardised all $\mathrm{M}$ variables. We report the indirect effect $(\mathrm{X} \rightarrow \mathrm{M} \rightarrow \mathrm{Y}$ ) as the main index of mediation.

The mediation analyses were conducted in $\mathrm{R}$ ( $\mathrm{R}$ Core Team, 2017) using the "mediation" package (Tingley, Yamamoto, Hirose, Keele \& Imai, 2014) with default arguments of the mediate function, which uses bootstrapping to determine significance (Imai, Keele \& Tingley, 2010). Data were resampled with replacement with 5,000 bootstraps, and the distribution of indirect, direct, and total effects were returned. We report the indirect effect estimates, their associated confidence intervals, and $p$ values in-text, with more detailed summaries of the models in the online supplementary materials (see https://osf.io/hs9me/). We also present Bayes Factors $(\mathrm{BF})$ to describe the likelihood of the data in the mediation or the null hypothesis (i.e., that there is no mediation effect). Using the R package "BayesMed" (Nuijten, Wetzels, Matzke, Dolan, \& Wagenmakers, 2015), we used the default settings of the $j z s$ med function to perform mediation largely as described above. This function uses a JeffreysZellner-Siow prior (Liang, Paulo, Molina, Clyde \& Berger, 2008) and takes 10,000 samples, with 500 "burn-in" samples using JAGS software (Hornik, Leisch \& Zeileis, 2003). We present the resulting $\mathrm{BF}_{10}$, which describes the evidence in favour of the mediation effect, and the inverse $\left(\mathrm{BF}_{01}\right)$ for a null mediation. As per Kass and Raftery (1995), Bayes factor values are referred to as positive $(>3)$, strong $(>20)$, and very strong $(>150)$ evidence for mediation $\left(\mathrm{B}_{10}\right)$ or for the null $\left(\mathrm{B}_{01}\right)$. Full descriptive output for both types of models can be found online (https://osf.io/hs9me/).

Significant mediation models were rerun with age as a possible moderator, using Hayes (2017) model 58, which includes an interaction with age on both predictor-mediator, and mediator-outcome paths. To test for significant moderated mediation, we compared conditional indirect effects for different values of age, selected to be the mean of each age group: Adolescent (16.8 yr), Young Adult (20.8 yr), and Adult (28.4 yr). If one group had a significant mediation effect and at least one other did not, we had some evidence for moderated mediation, i.e., mediation is dependent upon age. We tested whether the moderated mediation was significant using the test.modmed function in the "mediation" package. This tests the conditional indirect effects at one value of age against another and returns the beta value difference, $95 \%$ confidence interval, and $p$ value. Because the Bayesian analysis for moderation has not yet been implemented in the 'BayesMed' package, we could not calculate Bayes factors.

\section{Results}

\section{Age and Sex Effects on Predictor, Outcome, and Cognitive Control PCA Components}

Means and standard deviations are shown for males and females in each age group in Table 4. Predictor components showed a number of significant age and sex effects (Table 5A). The Adolescent group scored higher on the Delay Aversion and Impulsive Decisions PCA components, 
compared with both the Young Adults and Adult groups, indicating a tendency to respond faster and more impulsively, even though they did not report higher impulsivity or benefits of risk-taking (Impulsivity, Risk Propensity components). Yet, both Adolescent and Young Adult groups reported greater perceived benefits of antisocial behaviours compared to the Adult group (Work \& Aggression). Males reported higher benefits of risky behaviours (Risk Propensity) and benefits of antisocial behaviour (Work and Aggression), but their responding showed less impulsive choice (Impulsive Decisions) than females.

Outcome and Cognitive Control components showed no effects of Sex (Tables 5B and C). An Age effect was significant for both Psychological Distress and General-EF

Table 4 Age group means and standard deviations (SD) of the (A) Predictor (B) Outcome and (C) Cognitive Control PCA component scores, as well as the task-switching and BRIEF variables

\begin{tabular}{|c|c|c|c|c|c|c|c|c|c|c|c|c|}
\hline \multirow[b]{3}{*}{ Measure } & \multicolumn{4}{|c|}{ Adolescents } & \multicolumn{4}{|c|}{ Young adults } & \multicolumn{4}{|l|}{ Adults } \\
\hline & \multicolumn{2}{|l|}{ Male } & \multicolumn{2}{|l|}{ Female } & \multicolumn{2}{|l|}{ Male } & \multicolumn{2}{|l|}{ Female } & \multicolumn{2}{|l|}{ Male } & \multicolumn{2}{|l|}{ Female } \\
\hline & Mean & SD & Mean & SD & Mean & SD & Mean & SD & Mean & SD & Mean & SD \\
\hline \multicolumn{13}{|l|}{ A. Predictors Components } \\
\hline Risk Propensity & -0.044 & 0.981 & -0.230 & 1.035 & 0.581 & 0.932 & -0.279 & 0.841 & 0.653 & 0.957 & -0.282 & 0.947 \\
\hline Delay Aversion & 0.453 & 1.043 & 0.443 & 1.113 & -0.122 & 0.942 & -0.361 & 0.913 & -0.354 & 0.669 & -0.270 & 0.696 \\
\hline Impulsive Decisions & 0.191 & 0.868 & 0.408 & 0.947 & -0.284 & 0.995 & -0.097 & 1.044 & -0.749 & 0.823 & 0.063 & 0.995 \\
\hline Impulsivity & 0.128 & 1.254 & 0.269 & 1.012 & 0.004 & 0.859 & -0.241 & 0.813 & -0.379 & 0.668 & 0.003 & 1.167 \\
\hline Work and Aggression & 0.438 & 1.160 & -0.358 & 0.660 & 0.463 & 1.149 & -0.115 & 0.924 & -0.513 & 0.279 & -0.348 & 0.812 \\
\hline \multicolumn{13}{|l|}{ B. Outcomes Components } \\
\hline Psychological Distress & 0.141 & 1.015 & 0.443 & 1.310 & -0.038 & 0.907 & -0.255 & 0.671 & -0.520 & 0.785 & -0.120 & 0.972 \\
\hline Risky Behaviours & -0.151 & 0.810 & 0.005 & 1.159 & 0.369 & 1.100 & -0.198 & 0.863 & 0.208 & 0.900 & -0.125 & 1.002 \\
\hline Quality of Life & -0.024 & 1.164 & 0.163 & 0.966 & 0.053 & 0.892 & -0.149 & 0.965 & -0.215 & 1.288 & 0.052 & 0.982 \\
\hline \multicolumn{13}{|c|}{ C. Cognitive Control Components } \\
\hline General-EF & 0.412 & 1.039 & 0.603 & 0.938 & -0.254 & 0.905 & -0.277 & 0.725 & -0.121 & 1.225 & -0.482 & 0.981 \\
\hline Working Memory Span & -0.052 & 0.941 & -0.202 & 1.008 & 0.225 & 1.213 & -0.095 & 0.993 & 0.428 & 0.863 & 0.006 & 0.709 \\
\hline \multicolumn{13}{|l|}{ BRIEF } \\
\hline Behavior Regulation & 54.3 & 10.7 & 55.1 & 12.5 & 49.4 & 9.3 & 49.2 & 8.1 & 46.4 & 6.6 & 51.5 & 11.9 \\
\hline Metacognition & 56.6 & 12.2 & 57.7 & 12.2 & 50.8 & 8.5 & 50.2 & 7.6 & 50.4 & 8.0 & 53.0 & 12.6 \\
\hline \multicolumn{13}{|l|}{ Task-switching Behaviour } \\
\hline All-repeat RT & 596 & 180 & 583 & 93.5 & 525 & 60 & 538 & 56 & 584 & 84 & 533 & 69 \\
\hline Mixed-repeat $R T$ & 672 & 197 & 661 & 160.4 & 593 & 110 & 594 & 70 & 670 & 112 & 599 & 88 \\
\hline Switch $R T$ & 903 & 515 & 862 & 276.4 & 733 & 247 & 701 & 176 & 815 & 176 & 707 & 193 \\
\hline All-repeat Error Rate & 2.56 & 2.15 & 3.09 & 1.88 & 2.63 & 2.89 & 1.63 & 1.39 & 2.47 & 1.46 & 1.82 & 1.78 \\
\hline Mixed-repeat Error Rate & 3.28 & 2.97 & 3.55 & 3.14 & 2.39 & 2.39 & 1.71 & 1.81 & 1.80 & 1.73 & 2.04 & 2.56 \\
\hline Switch Error Rate & 5.95 & 6.15 & 7.67 & 6.41 & 4.99 & 5.61 & 3.15 & 2.80 & 5.02 & 4.15 & 4.59 & 5.01 \\
\hline \multicolumn{13}{|l|}{ Task-switching ERPs } \\
\hline Early Mixing Positivity & 28.8 & 13.5 & 24.5 & 11.1 & 20.9 & 8.2 & 23.7 & 10.5 & 15.6 & 5.8 & 18.7 & 8.9 \\
\hline Late Mixing Positivity & 34.0 & 17.5 & 30.1 & 13.6 & 25.5 & 11.3 & 29.8 & 16.3 & 17.8 & 9.2 & 21.7 & 11.5 \\
\hline Early Switch Positivity & 30.0 & 16.5 & 28.0 & 12.1 & 26.2 & 10.9 & 26.7 & 12.1 & 24.6 & 8.5 & 27.0 & 11.7 \\
\hline Late Switch Positivity & 32.8 & 13.2 & 32.5 & 15.8 & 25.7 & 10.8 & 28.3 & 11.5 & 21.7 & 11.2 & 27.1 & 10.9 \\
\hline \multicolumn{13}{|l|}{ Task-switching MVPA } \\
\hline Max Class - Mixing & 58.61 & 6.42 & 56.11 & 3.67 & 55.99 & 3.47 & 56.39 & 4.13 & 57.18 & 2.80 & 56.55 & 3.06 \\
\hline Max Class - Switch & 56.63 & 3.61 & 56.66 & 3.74 & 56.61 & 4.52 & 57.09 & 3.89 & 56.91 & 3.79 & 56.33 & 3.68 \\
\hline
\end{tabular}

For all components, high scores indicate poorer performance (e.g., greater Risk Propensity, greater Psychological Distress, poorer General-EF. EF = Executive Function. 
components, indicating that Adolescents reported poorer psychological wellbeing and performed more poorly on cognitive control tasks than both Young Adults and Adults. These findings are consistent with the significant Age effect on the BRIEF Behavioural Regulation and Metacognition Indices (BRI: $F_{(2,156)}=27.72, p<0.0001,{ }_{\mathrm{p}} \eta^{2}=0.26$; MI: $F_{(2,156)}=$ 14.03, $\left.p<0.0001,{ }_{\mathrm{p}} \eta^{2}=0.15\right)$, where Adolescents reported lower cognitive control than both Adult and Young Adult groups (BRI: $p<0.0001, p=0.001$; MI: both $p<0.0001$ ).

\section{Task-switching}

Behavioural Data Both median RT and percent errors (Figure 3) showed significant main effects of Trial Type (Table 6). There was a significant mixing cost for RT (69 $\mathrm{ms}$ ) but not error rate, whereas switch cost was significant for both RT (156 ms) and error rate $(2.67 \%)$.

There was a significant Age effect on both RT and error rate, with the Adolescent group responding more slowly than Young Adults and less accurately than both Young Adults and Adults. While the Trial Type x Age interaction was significant for both RT and error rate, only RT switch cost was significantly larger for Adolescents compared to Adults.

ERPs Figure 4A shows ERP waveforms over the cue-target interval (CTI) for each trial type at three posterior midline sites where differences were most pronounced. The scalp distribution of early and later sections of the mixing- and switchpositivity are shown in Figure 4B. Outcomes of statistical analyses are shown in Table 6.

Mixing-positivity All-repeat and repeat trials differentiated after 250 ms with a "mixing-positivity" for repeat trials emerging across the N2/P3 period, and extending posteriorly to the end of the CTI, with a reverse late effect evident more anteriorly (Figure 4). The amplitude of both early (mean latency: $373 \pm 55 \mathrm{~ms}$; mean amplitude: $23 \pm 11 \mu \mathrm{V} / \mathrm{cm}^{2}$ ) and the late $\left(652 \pm 134 \mathrm{~ms} ; 28 \pm 15 \mu \mathrm{V} / \mathrm{cm}^{2}\right)$ sections of the mixingpositivity varied significantly across Age groups, with larger early and late mixing positivity in Adolescents and Young Adults compared with Adults. Adolescents also had a larger early mixing positivity compared to Young Adults.

Switch-positivity Differentiation between repeat and switch trials emerged first over the posterior $\mathrm{P} 2$, and then again before N2 onset, resolving by the end of the P3 (Figure 4). The early section of the switch-positivity $(283 \pm 24 \mathrm{~ms} ; 27 \pm 12$ $\mu \mathrm{V} / \mathrm{cm}^{2}$ ) was laterally and parietally distributed (Figure 4B) and did not vary with Age. The late switch-positivity (433 \pm $62 \mathrm{~ms} ; 29 \pm 13 \mu \mathrm{V} / \mathrm{cm}^{2}$ ) was more clearly defined centroparietally and was larger for Adolescents than Young Adults and Adults.
MVPA Figures 5 shows the outcomes from MVPA analyses for mixing cost and switch cost ${ }^{2}$. Peak differentiation between repeat and all-repeat trials and between switch and repeat trials occurred before target onset in $96 \%$ and $95 \%$ of participants, respectively.

The average classification rate for mixing cost peaked around $200 \mathrm{~ms}$ at just greater than 55\% correct and differed significantly from chance between $100 \mathrm{~ms}$ and $800 \mathrm{~ms}$ postcue (Figure 5Ai). Maximum classification rate varied from $50 \%$ to $75 \%$, with a mean of $57 \%$ around $300 \mathrm{~ms}$ post-cue (Figure 5Bii \& iii). For switch cost (Figure 5B), the average classification rate peaked around $250 \mathrm{~ms}$ at just over $55 \%$ correct and differed significantly from chance between $150 \mathrm{~ms}$ and $700 \mathrm{~ms}$ post-cue (Figure 5Bi). The average maximum classification rate for switch cost also was $57 \%$ at around $300 \mathrm{~ms}$ (Figures 5Bii and iii). There were no effects of Age group or sex on maximum classification rate (Table 6).

\section{Associations between predictors, outcomes, and cognitive control mediators}

Pearson correlations (uncorrected) between predictor, outcome, and cognitive control measures were used to identify variables to enter into the mediation model (Table 7). Correlations between all measures are shown in online at https://osf.io/hs9me/.

Predictor and outcome relationships $(X \rightarrow Y)$ The two predictor components that had high loadings from self-report measures correlated moderately to strongly with outcome components, which all relied on self-report. Not surprisingly, given that they have high loadings from different sections of the same questionnaire (CARE), the Risk Propensity component strongly correlated with the Risky Behaviours component. The Impulsivity component positively correlated with all three outcomes components. Note that the two predictor components that had high loadings from behavioural scores did not correlate with any of the outcome components.

\footnotetext{
${ }^{2}$ Based on individually estimated null classification rates (using $p$ value $<$ 0.05 , uncorrected), the peak classification score of some participants did not differ significantly from chance. Specifically, $10 \%(\mathrm{~N}=17)$ did not significantly discriminate switch and repeat trials and $20 \%(\mathrm{~N}=36)$ did not significantly discriminate all-repeat and repeat trials (47 participants in total). To determine whether "discriminators" $(\mathrm{N}=121)$ and "nondiscriminators" $(\mathrm{N}=$ 47) differed in any way that may impact further analyses, we compared the two groups using independent samples $t$-tests on all other variables using FDR (alpha $<0.05$ ) corrected $t$-tests. The groups did not differ on any MVPA, behavioural, or ERP task-switching measures, nor did they differ by age, sex, or any predictor, outcome, and cognitive control variable $(p=0.168-$ 0.979). Excluding the 47 "nondiscriminators" did not affect the MVPA analyses $(p=0.173-0.793)$. Consequently, all further analyses were conducted on the entire sample of 168 participants.
} 
Table 5 Outcomes of age group x sex ANOVA for (A) Predictor (B) Outcome and (C) Cognitive control PCA component scores

\begin{tabular}{|c|c|c|c|c|c|c|}
\hline COMPONENTS & Factor & $\mathrm{F}$ & $d f$ & $p$ & ${ }_{p} \eta^{2}$ & Direction of significant effects \\
\hline \multicolumn{7}{|l|}{ A. Predictors } \\
\hline \multirow[t]{3}{*}{ Risk propensity } & Age & 1.888 & 2,162 & 0.155 & 0.023 & \\
\hline & Sex & 16.045 & 1,162 & $<0.0001$ & 0.090 & $\mathbf{M}>\mathbf{F}^{* *}$ \\
\hline & Age*Sex & 2.581 & 2,162 & 0.079 & 0.031 & \\
\hline \multirow[t]{3}{*}{ Delay aversion } & Age & 10.617 & 2,162 & $<0.0001$ & 0.116 & AG1 $>$ AG $2 * *$, AG1 $>$ AG3 $* *$ \\
\hline & Sex & 0.110 & 1,162 & 0.740 & 0.001 & \\
\hline & Age*Sex & 0.386 & 2,162 & 0.680 & 0.005 & \\
\hline \multirow[t]{2}{*}{ Impulsive decisions } & AgeSex & $\begin{array}{l}5.926 \\
5.761\end{array}$ & $\begin{array}{l}2,162 \\
1,162\end{array}$ & $\begin{array}{l}0.003 \\
0.018\end{array}$ & $\begin{array}{l}0.068 \\
0.034\end{array}$ & $\begin{array}{l}\text { AG1 }>\text { AG2*, AG1 }>\text { AG3* } \\
\text { M }<\mathrm{F}^{*}\end{array}$ \\
\hline & Age*Sex & 1.084 & 2,162 & 0.341 & 0.013 & \\
\hline \multirow[t]{3}{*}{ Impulsivity } & Age & 2.206 & 2,162 & 0.113 & 0.027 & \\
\hline & Sex & 0.286 & 1,162 & 0.593 & 0.002 & \\
\hline & Age*Sex & 1.189 & 2,162 & 0.307 & 0.014 & \\
\hline \multirow[t]{3}{*}{ Work and aggression } & Age & 3.948 & 2,162 & 0.021 & 0.046 & AG1 $>$ AG3*, AG2>AG3* \\
\hline & Sex & 5.997 & 1,162 & 0.015 & 0.036 & $\mathbf{M}>\mathbf{F}^{*}$ \\
\hline & Age*Sex & 2.385 & 2,162 & 0.095 & 0.029 & \\
\hline \multicolumn{7}{|l|}{ B. Outcomes } \\
\hline \multirow[t]{3}{*}{ Psychological distress } & Age & 4.980 & 2,162 & 0.008 & 0.058 & AG1 $>$ AG2 $\uparrow$, AG1 $>$ AG3* \\
\hline & Sex & 0.900 & 1,162 & 0.344 & 0.006 & \\
\hline & Age*Sex & 1.594 & 2,162 & 0.206 & 0.019 & \\
\hline \multirow[t]{3}{*}{ Risky behaviours } & Age & 0.436 & 2,162 & 0.647 & 0.005 & \\
\hline & Sex & 2.051 & 1,162 & 0.154 & 0.012 & \\
\hline & Age*Sex & 2.253 & 2,162 & 0.108 & 0.027 & \\
\hline \multirow[t]{3}{*}{ Quality of life } & Age & 0.309 & 2,162 & 0.734 & 0.004 & \\
\hline & Sex & 0.230 & 1,162 & 0.632 & 0.001 & \\
\hline & Age*Sex & 0.852 & 2,162 & 0.429 & 0.010 & \\
\hline \multicolumn{7}{|l|}{ C. Cognitive Control } \\
\hline \multirow[t]{3}{*}{ General-EF } & Age & 13.579 & 2,162 & $<0.0001$ & 0.144 & AG1 $>$ AG2 $* *$, AG1 $>$ AG3 $* *$ \\
\hline & Sex & 0.159 & 1,162 & 0.690 & 0.001 & \\
\hline & Age*Sex & 0.820 & 2,162 & 0.442 & 0.010 & \\
\hline \multirow[t]{3}{*}{ Working memory span } & Age & 1.240 & 2,162 & 0.292 & 0.015 & \\
\hline & Sex & 2.913 & 1,162 & 0.090 & 0.018 & \\
\hline & Age*Sex & 0.208 & 2,162 & 0.812 & 0.003 & \\
\hline
\end{tabular}

For all components, high scores indicate poorer performance (e.g., greater Risk Propensity, greater Psychological Distress, poorer General-EF.

$\mathrm{EF}=$ Executive Function. For significant Age Group and Sex effects, simple comparisons show differences between Adolescent (AG1), Young Adults (AG2) and Adult (AG3) groups, as well as males (M) and females (F). Only significant contrasts are reported. $\dagger p<0.05 ; * p<0.01 ; * * p<0.001$.

Predictors and cognitive control relationships $(X \rightarrow M)$ The Impulsivity component (which consisted primarily of the BIS-11) correlated stronger with the BRIEF Behavioural Regulation and Metacognition indices and weaker with the General-EF and the WM Span components, as well as RT switch cost from the task-switching paradigm. The Impulsive Decisions component (which represents preference for fast choices without regard for strength of evidence from the IST) also correlated moderately with General-EF and weaker with the late switch-positivity amplitude. The Delay Aversion component (a behavioural measure which also taps into impulsive choices or a preference for fast decisions that do not optimise rewards from the CGT) correlated strongly with the General-EF component, RT switch cost, early and late switchpositivity amplitude, error mixing cost, as well as Behavioural Regulation and Metacognition BRIEF indices. Finally, the Work \& Aggression component correlated weakly with both RT switch cost and the BRIEF Behavioural Regulation index. Overall, this pattern of findings indicates that participants who reported being impulsive and who showed evidence of impulsive choices and aversion to slow and considered decisionmaking performed more poorly on tasks that require cognitive control, showed more effortful preparation to set-shift, and reported poorer levels of cognitive control. 

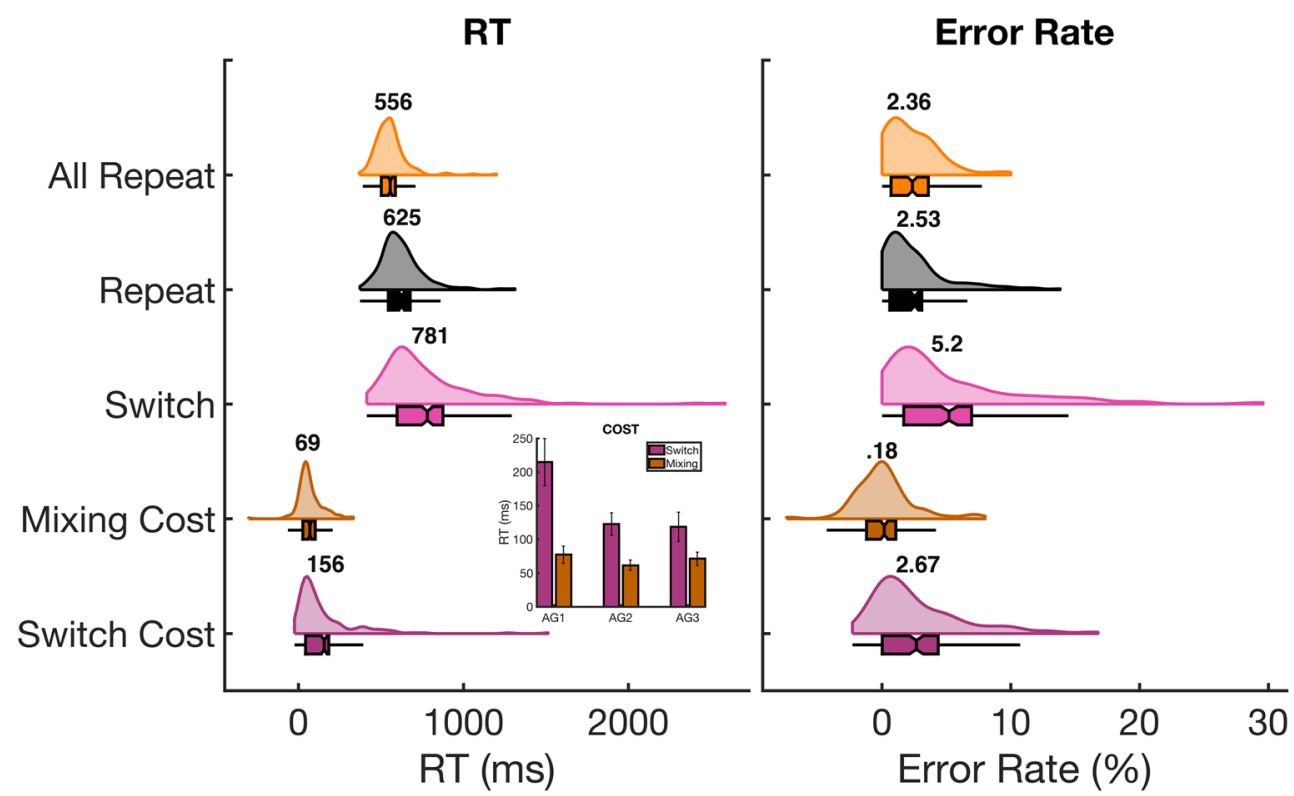

Fig. 3. Behavioural task-switching measures. Left: Median RT (ms). Right: Error rate (percentage) for each trial type and mixing/switch costs from the task-switching paradigm. Plots display the distribution of each

data series with a superimposed box and whisker plot. Notch centre is the mean score, box edges $=1^{\text {st }}$ and $3^{\text {rd }}$ quartile, whisker ends $= \pm 1.5$ interquartile range. Numbers represent group averages

Cognitive control and outcome relationships $(M \rightarrow Y)$ There were no significant correlations between task switching measures of cognitive control and outcome components. The General-EF component was only weakly correlated with the Psychological Distress component, and unlikely to survive correction for multiple comparisons. In contrast, BRIEF indices correlated with all three outcome components, and most strongly with Psychological Distress. So, self-report measures of cognitive control, but not behavioural measures of cognitive control, correlated with self-report measures of psychological wellbeing, engagement in risky behaviours, and quality of life.

\section{Mediation and Moderation analyses}

Mediation models were run only for significant paths between predictor and outcome components (Table 7). For consistency, all cognitive control variables were entered as mediators, irrespective of whether they significantly correlated with predictor and/or outcome components. Cognitive control mediators included the two cognitive control components extracted from PCA (General-EF, WM-Span), the Behaviour Regulation and Metacognition indices of the BRIEF, and both mixing cost and switch cost measures from the task-switching paradigm (RT, error rate, early-positivity, late-positivity, MVPA max classification rate). The full table of mediation results are presented in the supplementary materials (https://osf.io/hs9me/). We discuss the significant models and describe the Bayesian evidence for the null hypothesis (i.e., no mediation effect) where applicable.
BRIEF Indices as mediators Because the BRIEF indices showed the strongest correlations with both predictor and outcome components, we first examined them as mediators. Figure 6A shows that the relationship between Impulsivity and Psychological Distress $(\beta=0.527)$ was significantly mediated by both Metacognition (top) and Behaviour Regulation (bottom) indices, with strong Bayes Factors supporting these mediation effect. Specifically, the Impulsivity $\rightarrow$ Psychological Distress relationship was reduced by $\beta=0.22$ ([0.095, 0.353], $p_{\mathrm{FDR}}$ $\left.<0.001, \mathrm{BF}_{10}=106\right)$ when controlling for Metacognition and by $\beta=0.326\left([0.227,0.439], p_{\mathrm{FDR}}<0.001, \mathrm{BF}_{10}=\right.$ $2.13 \times 10^{11}$ ) when controlling for Behavioural Regulation. Although both mediation (indirect) effects were highly significant, in both cases the remaining Impulsivity $\rightarrow$ Psychological Distress relationship (direct effect) remained significant, indicating that self-report measures of cognitive control only partially mediate this relationship.

To examine whether age moderated the relationships in the above mediation models, we compared the mediation effect of each BRIEF Index at the mean age of each Age Group: Adolescents (16.8 yr), Young Adults (20.8 yr), and Adults (28.4 yr). The mediation (indirect) effect of both Metacognition and Behaviour Regulation Indices on the Psychological Distress $\rightarrow$ Impulsivity relationship was significant for each group, indicating that age does not significantly moderate the above mediation effect (for MI and BRI, respectively: Adolescents: $\beta=0.207$ [0.097, $0.32], p \leq 0.0001$, and $\beta=0.315[0.216,0.42], p \leq$ 
Table 6 Task-switching statistical results for (A) Behavioural, (B) ERP, (C) MVPA measures

\begin{tabular}{|c|c|c|c|c|c|c|}
\hline Variable & Factor & $d f$ & $\mathrm{~F}$ & $p$ & ${ }_{p} \eta^{2}$ & Direction of significant effects \\
\hline \multicolumn{7}{|l|}{ A. Behaviour } \\
\hline \multirow[t]{7}{*}{$R T$} & Trial & 2,324 & 110.7 & $<0.0001$ & 0.406 & Mixing Cost ${ }^{* *}$, Switch Cost ${ }^{* *}$ \\
\hline & Age & 2,162 & 5.988 & 0.003 & 0.069 & AG1>AG2** \\
\hline & Sex & 1,162 & 1.481 & 0.225 & 0.009 & \\
\hline & Trial*Age & 4,324 & 3.854 & 0.017 & 0.045 & Switch: $F_{(2,162)}=3.935, \mathrm{p}=.021,{ }_{p} \eta^{2}=.046 ;$ AG1 $>$ AG2 $*$ \\
\hline & Trial*Sex & 2,324 & 1.076 & 0.313 & 0.007 & \\
\hline & Sex*Age & 2,162 & 0.429 & 0.652 & 0.005 & \\
\hline & Trial*Sex*Age & 4,324 & 0.041 & 0.976 & 0.001 & \\
\hline \multirow[t]{7}{*}{ Error rate } & Trial & 2,324 & 56.283 & $<0.0001$ & 0.258 & Switch Cost** \\
\hline & Age & 2,162 & 5.378 & 0.005 & 0.062 & AG1>AG2*,AG1>AG3 $\dagger$ \\
\hline & Sex & 1,162 & 0.161 & 0.689 & 0.001 & \\
\hline & Trial*Age & 4,324 & 3.044 & 0.032 & 0.036 & Switch: $F_{(2,162)}=2.567, p=0.080,{ }_{p} \eta^{2}=0.031 ; \mathrm{AG} 1>\mathrm{AG} 2 \dagger$ \\
\hline & Trial*Sex & 2,324 & 0.136 & 0.873 & 0.001 & \\
\hline & Sex*Age & 2,162 & 1.987 & 0.140 & 0.024 & \\
\hline & Trial*Sex*Age & 4,324 & 1.362 & 0.256 & 0.017 & \\
\hline \multicolumn{7}{|l|}{ B. ERPs } \\
\hline \multirow{4}{*}{$\begin{array}{l}\text { Early mixing } \\
\text { Positivity }\end{array}$} & Age & 2,162 & 8.063 & 0.0004 & 0.091 & AG1 $>$ AG2 $\uparrow$, AG1 $>$ AG $3 * *, A G 2>A G 3 \dagger$ \\
\hline & & & & & & \\
\hline & Sex & 1,162 & 0.068 & 0.794 & $<0.001$ & \\
\hline & Age*Sex & 2,162 & 2.252 & 0.108 & 0.027 & \\
\hline \multirow{4}{*}{$\begin{array}{l}\text { Late mixing } \\
\text { Positivity }\end{array}$} & Age & 2,162 & 6.944 & 0.001 & 0.079 & AG1 $>$ AG3**,AG2>AG3 $\uparrow$ \\
\hline & & & & & & \\
\hline & Sex & 1,162 & 0.342 & 0.559 & 0.002 & \\
\hline & Age*Sex & 2,162 & 1.546 & 0.216 & 0.019 & \\
\hline Early switch & Age & 2,162 & 0.927 & 0.398 & 0.011 & \\
\hline \multirow[t]{2}{*}{ Positivity } & Sex & 1,62 & 0.017 & 0.896 & $<0.001$ & \\
\hline & Age*Sex & 2,162 & 0.337 & 0.715 & 0.004 & \\
\hline Late switch & Age & 2,162 & 5.231 & 0.006 & .061 & AG1>AG2*,AG1>AG3* \\
\hline \multicolumn{7}{|l|}{ Positivity } \\
\hline & Sex & 1162 & 1.411 & 0.237 & 0.009 & \\
\hline & Age*Sex & 2162 & 0.507 & 0.604 & 0.006 & \\
\hline \multicolumn{7}{|l|}{ C. MVPA } \\
\hline \multirow[t]{3}{*}{ Max Class - Mixing } & Age & 2162 & 1.298 & 0.276 & 0.016 & \\
\hline & Sex & 1162 & 1.527 & 0.218 & 0.009 & \\
\hline & Age*Sex & 2162 & 1.978 & 0.142 & 0.024 & \\
\hline \multirow[t]{3}{*}{ Max Class - Switch } & Age & 2162 & 0.059 & 0.943 & 0.001 & \\
\hline & Sex & 1162 & 0.001 & 0.977 & $<0.001$ & \\
\hline & Age*Sex & 2162 & 0.183 & 0.833 & 0.002 & \\
\hline
\end{tabular}

Mixing Cost $=$ Repeat - All-repeat, Switch Cost= Switch - Repeat. Max Class $=$ Maximum Classification Rate. $M=$ Male, $F=$ Female. For significant Age Group and Sex effects, simple comparisons show differences between Adolescent (AG1), Young Adults (AG2) and Adult (AG3) groups, as well as males (M) and females (F). For Trial*Age interaction, ANOVAs were conducted on switch and mixing costs to compare the three age groups. $\dagger p<0.05$; $* p<0.01 ; * * p<0.001$ (uncorrected). Only significant contrasts are reported.

0.0001 ; Young Adults: $\beta=0.205[0.096,0.32], p=$ 0.0004 , and $\beta=0.316[0.22,0.42], p \leq 0.0001$; Adults: $\beta=0.206[0.09,0.33], p=0.0004$, and $\beta=.314[.216$, $.42], p \leq 0.0001)$.
The relationship between Impulsivity $\rightarrow$ Quality of Life ( $\beta=0.414)$ was significantly reduced by $\beta=0.118$ ([0.024, $\left.0.208], p_{\mathrm{FDR}}=0.021, \mathrm{BF}_{10}=1.23\right)$, when Behavioural Regulation index was included as a mediator (Figure 6B). 


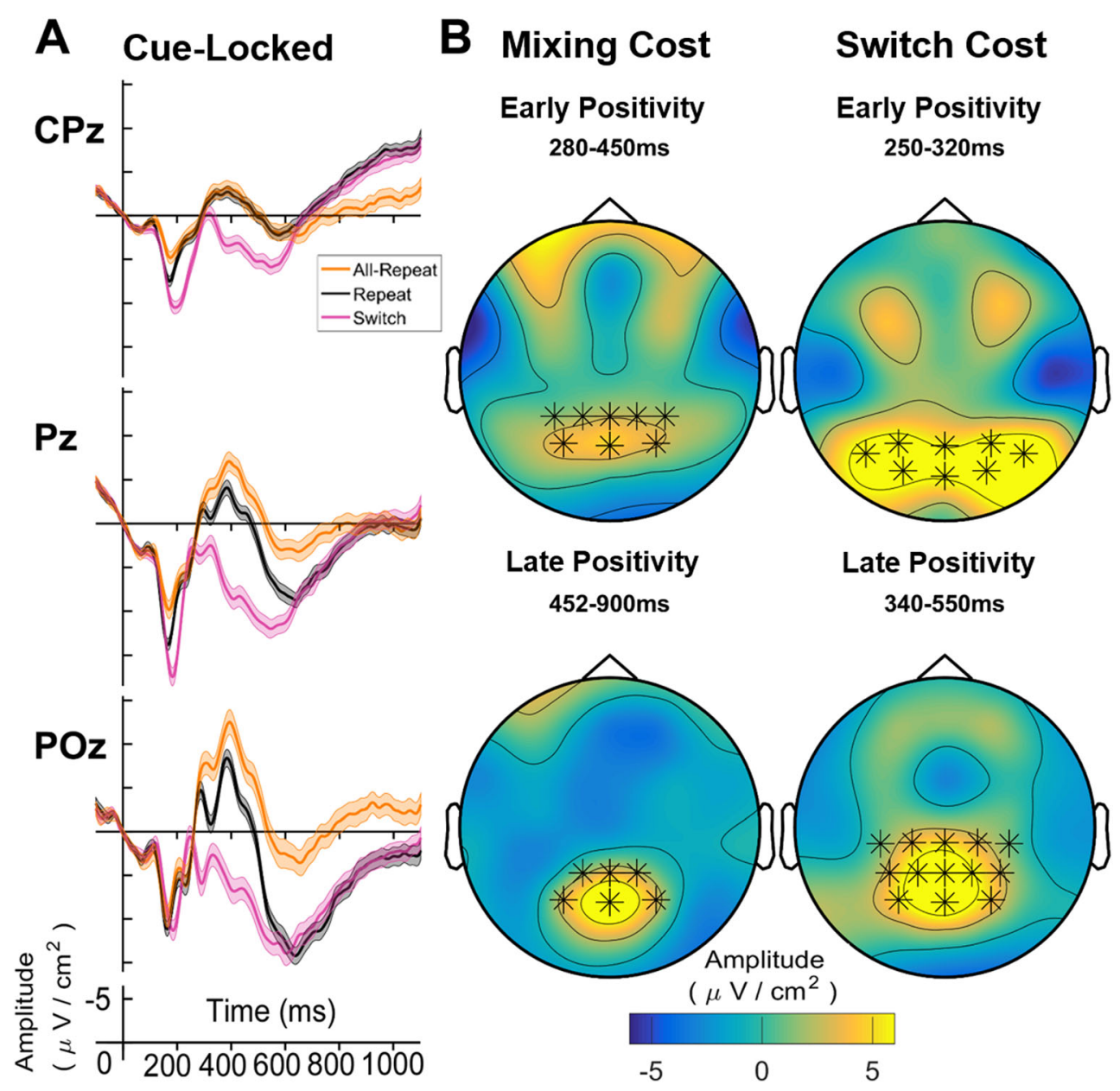

Fig. 4. Task-switching ERPs. (A) Cue-locked ERP average waveforms (with standard error) at three midline sites for each trial type. (B) Scalp distribution of cue-locked switch positivity and mixing positivity. Black stars on the headplots represent the electrodes included in the hotspot

Although statistically significant, this mediation effect was weak and not supported by the Bayes factor. Age did not significantly moderate this relationship (Adolescents $(\beta=0.117[0.021,0.22], p=0.014)$, Young Adults $(\beta=0.118[0.021,0.22], p=0.014)$, Adults $(\beta=0.117$ $[0.018,0.22], p=0.018)$.

The Behavioural Regulation index also significantly mediated the relationship between Work \& Aggression and Psychological Distress, $\beta=0.156$ ([0.042, 0.259], $p_{\mathrm{FDR}}=$ $0.041, \mathrm{BF}_{10}=4.98$. Although the mediation effect was weak and only weakly support by Bayesian analysis, the direct effect was no longer significant (Figure 6C) indicating a full mediation effect. Again, age did not moderate this mediation effect (Adolescents, $\beta=0.143$ [0.041, 0.25], $p=0.008$; Young Adults, $\beta=0.143[0.04,0.25], p=0.007$; Adults, $\beta=0.14$ $[0.038,0.25], p=0.009)$.

All remaining BRIEF mediation models had $\mathrm{BF}_{01}<16$, indicating weak evidence of mediation.
Other cognitive control variables as mediators No other cognitive control variable significantly mediated any relationship between predictor and outcome components. The exact evidence for the null in each model can be found at https://osf.io/hs $9 \mathrm{me} /$. There was between 20 and 50 times more evidence for the null in the vast majority of mediation models (57/70); 15 of those models showed at least 150 times more evidence for the null.

\section{Discussion}

This study examined whether variability in cognitive control ability mediates the relationship between level of impulsivity/reward processes and maladaptive outcomes in adolescence and young adulthood. We first discuss age and sex effects, and the relationships between 

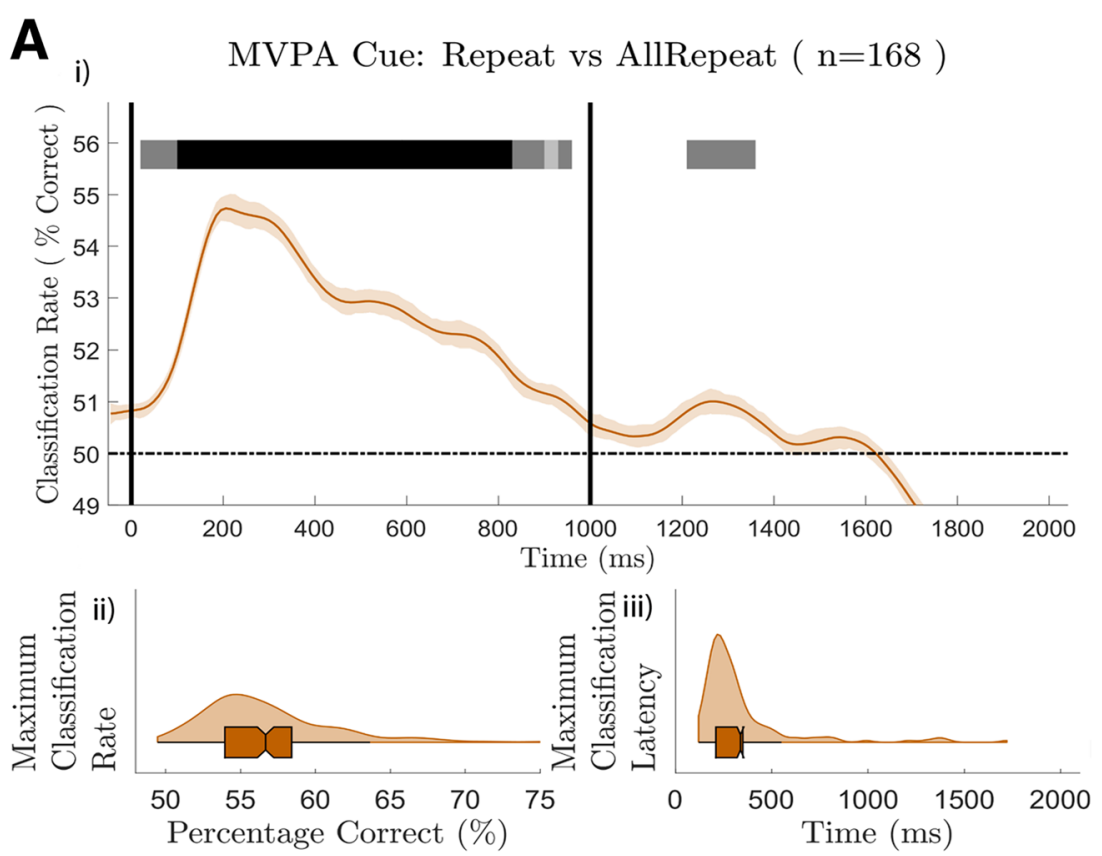

B MVPA Cue: Switch vs Repeat $(\mathrm{n}=168)$
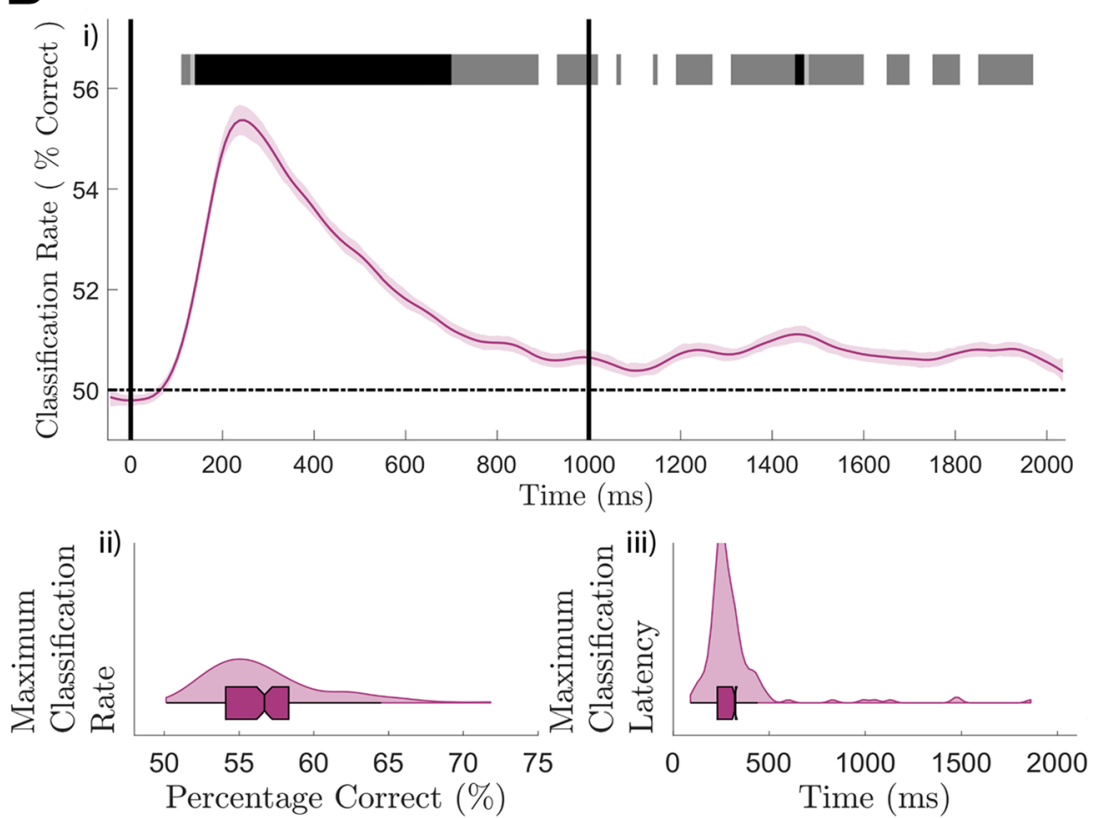

Fig. 5. MVPA outcomes for switch cost (switch vs. repeat trials; A) mixing cost (repeat vs. all-repeat trials; B). Mean classification rate waveform are shown for switch cost (Ai) and mixing cost (Bi) spanning from $50 \mathrm{~ms}$ pre-cue to $1,000 \mathrm{~ms}$ post-target. Aii (switch cost) and Bii (mixing

cost) show the distribution of the maximum classification rate, with the latency of the maximum classification shown in Aiii (switch cost) and Biii (mixing cost)

impulsivity/reward-related behaviours, outcome components tapping into risky behaviours and wellbeing, and cognitive control ability. We then discuss the outcomes of the mediation analyses in the context of risk-taking

models. Finally, we consider the implications of the different findings arising from self-report and behavioural measures purportedly tapping into the same constructs. 


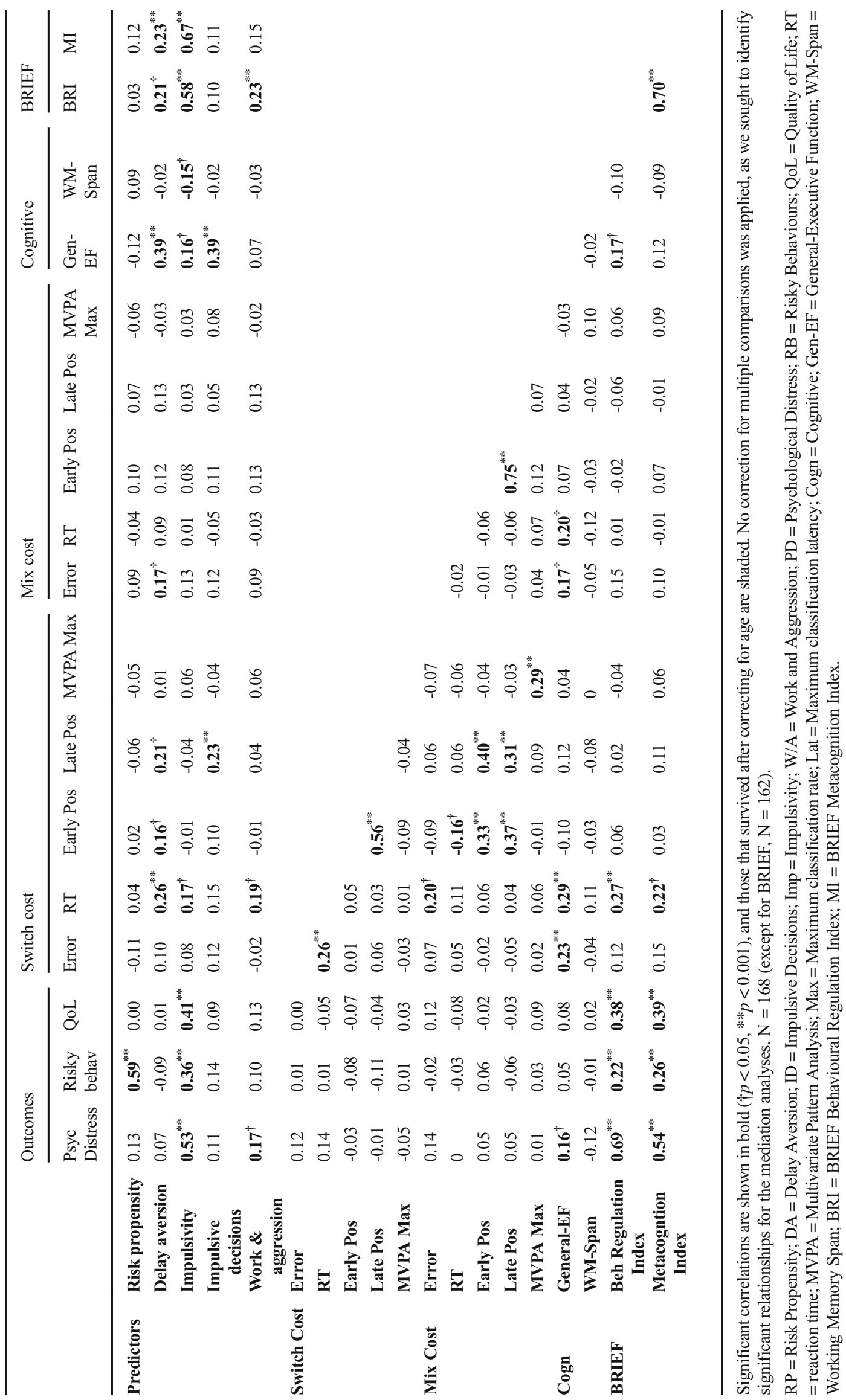




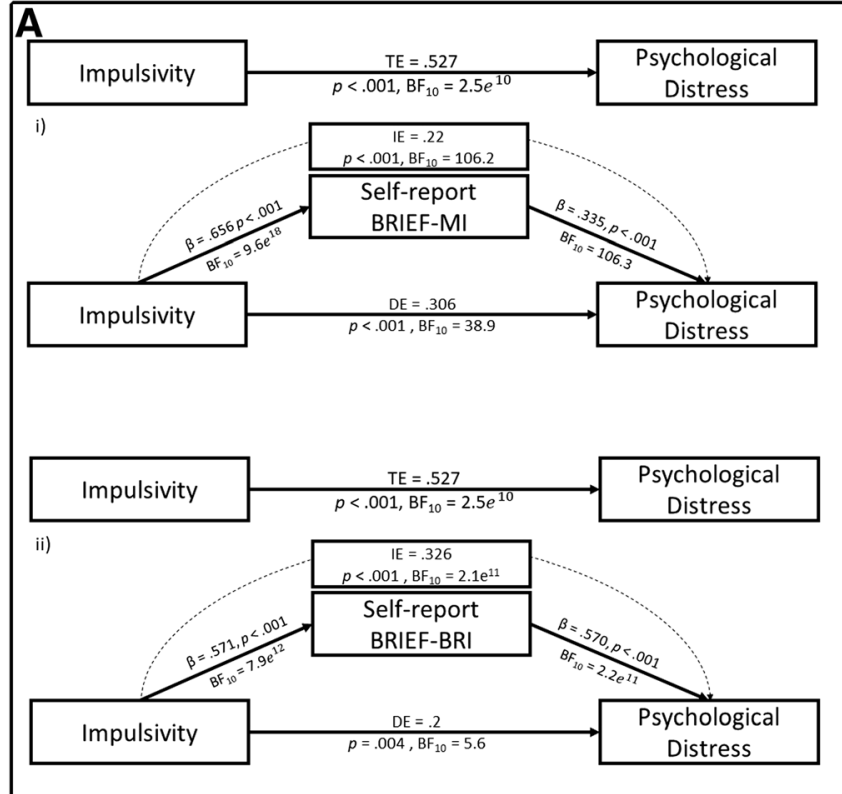

Fig. 6. Graphical summary of the significant mediation models. Description of the Total Effect (TE), Indirect Effect (IE), and Direct Effect (DE) of (A) self-report BRIEF Metacognition (Ai) and Behavioural Regulation (Aii) indices on the relationship between Impulsivity and Psychological Distress components, (B) self-report BRIEF Behavioural Regulation indices on the relationship between

\section{Effect of Age and Sex on Predictors, Outcomes, and Cognitive Control}

Adolescents performed more poorly than adults across a number of levels, whereas there were few differences between young adults and adults. ${ }^{3}$ Specifically, adolescents showed greater difficulty regulating behaviour to optimise outcomes and reported higher benefits of antisocial behaviours specifically related to work conduct and aggression (e.g., getting into a fight, being truant from school/work). Although they did not differ significantly from adults in self-reported level of quality of life or engagement in risky and antisocial behaviours (eg., alcohol, drugs), adolescents reported higher levels of psychological distress and poorer behaviour regulation and metacognition. This could suggest an awareness that they are not yet proficient in controlling their behaviour and using experience to guide future actions or result from the fact that these measures share common variance (see Do self-report and task-based measures tap into the same underlying constructs?). Adolescents also performed more poorly than adults on behavioural and electrophysiological measures of cognitive control, showing poorer reasoning, cognitive flexibility, planning, and

\footnotetext{
${ }^{3}$ Given the sparse and weak differences between young adult and adult groups (higher Work \& Aggression component and larger mixing positivity amplitude), they require replication before further interpretation.
}

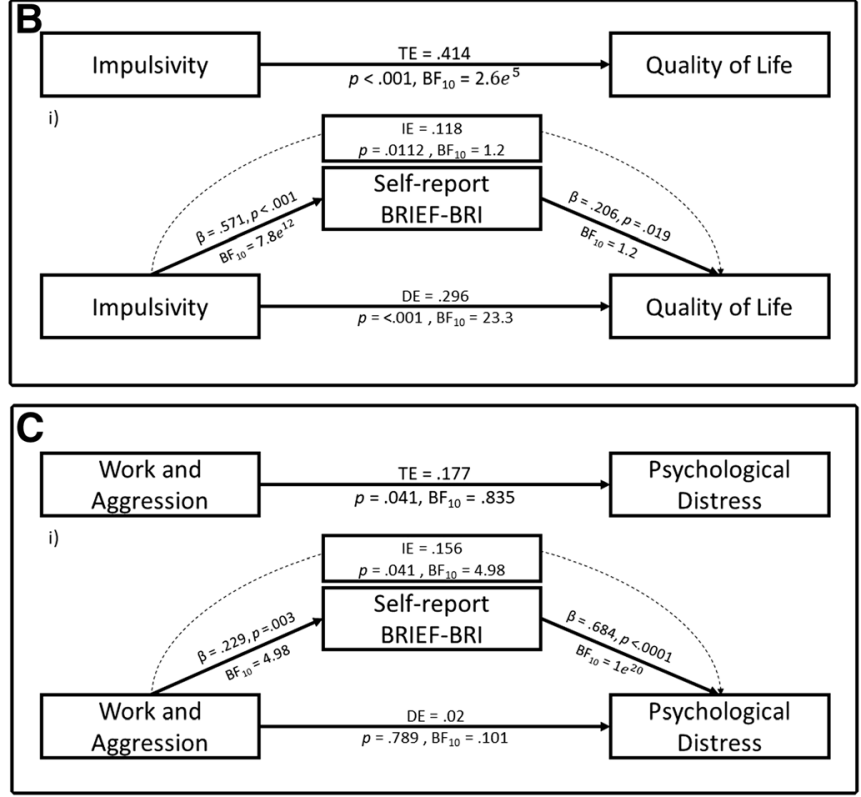

Impulsivity and Quality of Life components, and (C) self-report BRIEF Behavioural Regulation indices on the relationship between Work \& Aggression and Psychological Distress components. The standardized Beta value for each path/effect is presented, with associated $p$ value and Bayes factor $\left(\mathrm{BF}_{10}\right)$

more effortful and less efficient proactive control processes. Effects of sex were few and not entirely consistent (e.g., men reported higher benefits from risky behaviours, but showed better ability to control impulsive choices to adjust for risk than females) and did not interact with age.

This pattern of findings is consistent with adolescence as a period of continuing development of cognitive control processes, with difficulties regulating behaviour, especially in reward-related and/or emotional contexts (Casey, Jones, Hare, 2008; Stanford, Greve, Boudreaux, Mathias \& Brumbelow, 1996; Steinberg et al., 2008). The continued development of cognitive control processes from adolescence into adulthood is well documented (Luna, Garver, Urban, Lazar \& Sweeney, 2004; Ridderinkhof, Band \& Logan, 1999; Sander, Lindenberger \& Werkle-Bergner, 2012). Adolescents (and children) appear to require greater activation of cognitive resources and associated brain networks to perform a demanding cognitive task at the same or lower level than adults (Karayanidis, Jamadar \& Sanday, 2013; Blakemore \& Choudhury, 2006; Coch \& Gullick, 2012)-a type of developmental equivalent of the compensation-related utilisation of neural circuits hypothesis (CRUNCH; ReuterLorenz \& Cappell, 2008). This may result in inefficient implementation of cognitive control processes, especially when the emotional stakes are high (Diamond, 2013). Importantly, in relation to this study, the observed age effects are consistent with risk-taking models' profile of adolescents showing 
continuing maturation of cognitive control and reward-related processes, as well as higher levels of psychological distress.

\section{Relationships between Predictors, Outcomes, and Cognitive Control}

Higher levels of (self-reported) impulsivity were moderately to strongly correlated with higher psychological distress, greater engagement in risky behaviours, and poorer quality of life (outcome PCA components), as well as with poorer self-reported cognitive control, but only weakly with taskbased (behavioural and electrophysiological) measures of cognitive control. In contrast, task-based measures of impulsive decision-making and difficulty optimising reward were not associated with any outcomes components but did correlate with both task-based and self-report measures of cognitive control. These findings are consistent with previous evidence that self-report and behavioural measures of impulsivity are not robustly associated and that only the former correlate with self-reported level of wellbeing and risk-taking (Skippen et al., 2019; for review see Cyders \& Coskunpinar, 2011; Sharma, Markon \& Clark, 2014; Stahl et al., 2014; for further discussion, see Do self-report and task-based measures tap into the same underlying constructs?).

A similar pattern of relationships was found for the selfreport outcome components; they correlated moderately strongly with the self-report BRIEF indices but not with task-based measures of cognitive control. Thus, contrary to the predictions of risk-taking models, the association between cognitive control measures and either predictor or outcome components was weak, except amongst measures derived from the same level of analysis (i.e., exclusively self-report or exclusively behavioural).

In contrast to self-reported impulsivity, neither self-report measures of reward-seeking behaviours nor task-based measures of controlling impulsive choices to optimise reward significantly correlated with any outcome component. ${ }^{4}$ This is unlikely to be due to a measurement error (e.g., low variance), because many of these measures did show significant effects of age and/or sex, and controlling for age did not change the pattern of correlations. This finding is surprising, because contrary to the basic tenet of risk-taking models, one's ability to control impulsive choices to optimise reward was not related to their current level of risk-taking or maladaptive outcomes. Because current attitudes and behaviour are likely to have a

\footnotetext{
${ }^{4}$ The only exception was the significant correlation between perceived risk benefit (predictor) and level of engagement in risky behaviours (outcome) components, which have strong loadings from different forms of the same questionnaire (self-report CARE). This relationship was not mediated by self-report or task-based measures of cognitive control or vary with age. It is likely to be partially due to smearing of constructs across the two forms of the questionnaire (how much benefit from vs. how often do you engage in) and is not discussed further.
}

stronger impact on future outcomes (Bø, Billieux, Gjerde, Eilertsen \& Landrø, 2017; Moffitt et al. 2011), it is possible that these relationships will emerge more strongly in longitudinal perspectives.

\section{Does cognitive control ability mediate the relationship between impulsivity/perceived risk benefit and maladaptive outcomes?}

Mediation analyses showed that, consistent with our prediction, the effect of self-reported impulsivity on level of psychological distress was partially mediated by level of behaviour regulation and metacognitive ability derived from the BRIEF. A similar but weaker partial mediation effect was found for behaviour regulation on the effect of impulsivity, as well as the perceived benefits of antisocial work behaviour and aggression, on quality of life. Note that, again, all variables in this significant mediation model were derived (almost) exclusively from self-report instruments. In contrast, behavioural (or EEG) cognitive control measures derived from neuropsychological and experimental tasks did not mediate the significant relationships between self-reported impulsivity and any outcome component.

The correlations between impulsivity/reward-related predictor components, outcome components, and cognitive control variables remained for the most part significant when controlling for age (Table 7). Therefore, although adolescents show continuing development of processes related to rewardseeking, impulsive choices, cognitive control, and psychological distress, the intercorrelations between these variables remain largely stable across the adolescent to adult age range. Moreover, age did not significantly moderate any of the above mediation effects: self-reported level of cognitive control mediated the relationship between impulsivity and both psychological distress and quality of life equally across the adolescent to adult age range. This finding is inconsistent with the strong prediction of dual systems models that higher risktaking and greater incidence of maladaptive outcomes in adolescents are due to differences in relative rate of maturation of reward-related and cognitive control-related networks. It questions the key premise that adolescents engage in more risks because of protracted development of neural networks that support behaviour regulation versus reward mechanisms (Romer et al., 2017). Rather, the fact that this mediation effect was present independently of age suggests that variability in experiences gained through exploration, rather than age per se, can better account for individual variability in behaviour regulation and risk-taking behaviours across the lifespan (Romer et al. 2017). However, it is important to note that the absence of significant moderating effects of age in our data may have been influenced by low statistical power, as a result of dividing our sample into three age groups. 


\section{Do self-report and task-based measures tap into the same underlying constructs?}

Measures derived from self-evaluation more strongly predicted risk-taking and maladaptive outcomes than performance measures. Specifically, the Impulsivity component, based largely on the BIS-11, and the BRIEF indices correlated strongly with Psychological Distress but weaker with the other outcome components. Moreover, the BRIEF indices were the only significant mediators between Impulsivity and outcome components. At face value, these findings suggest that an individual's evaluation of their metacognitive ability and level of self-regulation is a more sensitive predictor than measures derived from their performance. However, there are a number of other, not mutually exclusive, alternative explanations.

Self-report and task-based (behavioural and ERP) measures may tap into different aspects of behaviour (McAuley, Chen, Goos, Schachar \& Crosbie, 2010). Across the board, self-report measures intercorrelated, irrespective of their designated status (i.e., predictors, mediators, or outcomes), and task-based measures also intercorrelated, albeit more weakly. Other studies also have reported that self-report and behavioural measures of cognitive control are not strongly correlated (e.g., stop-signal and Stroop tasks: Allom, Panetta, Mullan \& Hagger, 2016; neuropsychological measures: Burgess et al., 1998; Rabin et al., 2006). This could arise, at least partly, because self-report and task-based measures differ in the period of assessment: the former often require a retrospective assessment over a period ranging from weeks to months, whereas the latter assess average ability at the time of testing. While both may be influenced by situational variability (e.g., fatigue, boredom), self-report measures are likely to represent more stable, trait-like characteristics and task-based measures to be influenced more strongly by state variability (Sharma et al., 2014). Additionally, self-report measures typically request a broad evaluation covering a range of abilities as they impact everyday life (e.g., ability to plan ahead, evaluate costbenefits before engaging in risky behaviour), whereas taskbased measures target the implementation of distinct cognitive abilities in a "sanitised" lab environment (e.g., planning your strategy before initiating a response). Therefore, as suggested by McAuley et al. (2010), task-based assessments are sensitive to level of specific cognitive skills, whereas self-report assessments may evaluate the application of those skills and abilities in everyday life. The fact that correlations between different task-based measures were overall weak is consistent with the idea that task-based measures tend to assess distinct or minimally overlapping cognitive processes.

In addition, self-report measures are likely to tap into common sources of variance. An individual is likely to bring the same biases to the way they evaluate themselves in different self-report instruments (McAuley et al., 2010). Most obviously, current mood is very likely to impact one's perceived level of cognitive control efficiency, psychological well-being, and risk propensity. Likewise, one's perception of how well they regulate their behaviour at the current time may influence how they evaluate their level of impulsivity and engagement in risk-taking behaviours (e.g., one may evaluate having 5 drinks as risky or reasonable, depending on how much they feel in control over their decision to have them in that specific context). Moreover, self-report questionnaires tap into constructs that are not completely independent, such as impulsivity, behaviour regulation, emotion regulation, etc. The BRIEF indices were most strongly correlated with the Impulsivity predictor and Psychological Distress outcome components $(\mathrm{r}=0.54$ 0.69), which received strong loadings from the BIS-11 and the DASS, respectively. These questionnaires have several very similar items. For instance, items used to measure behaviour regulation in the BRIEF are largely identical to DASS items used to assess mood (e.g., I tend to overreact to situations vs. I overreact to small situations/problems, respectively), and the BRIEF scales correlate moderately to strongly with measures of anxiety and depression (Roth, Isquith \& Gioia, 2005). There also are many similar items in the BRIEF and the BIS-11 (e.g., I don't plan ahead for tasks vs. I plan tasks carefully, respectively) or even identical ones (e.g., I say things without thinking, is in both instruments).

Finally, the common variance between self-report measures of cognitive control and impulsivity could be partly related multidimensional structure of impulsivity (Dawe \& Loxton, 2004; Gullo \& Dawe, 2008). Specifically, the disinhibition component is more likely (than the reward sensitivity component) to be related to the cognitive control construct of response inhibition, and hence perceived ability to regulate one's behaviour, consistent with the conceptualisation of impulsivity by some models of risk-taking (e.g., Steinberg, Casey). However, in this case, we would have expected the behavioural measures of impulsivity derived from the CANTAB Gambling Task and Information Sampling Task to be more strongly associated with the self-regulation index of the BRIEF and behavioural/electrophysiological measures of interference control from the task-switching paradigm, which was not the case. We conclude that the multidimensional structure of impulsivity can't account for the difference in relationships between self-report and behavioural measures. In addition, these data show that, while some aspects of impulsivity may map directly onto difficulty in response inhibition, impulsivity is not fully encapsulated within or identifiable as a measure of cognitive control.

These findings caution against using single level of analysis (self-report or behavioural) or single measures at each level of analyses when seeking to quantify individual variability in cognitive control ability, its relationship to impulsivity and reward-related processes, and its impact on adaptive/ maladaptive behaviours. They also emphasise the need for better-defined key constructs to reduce common variance 
across self-report measures of different constructs and improve mapping between self-report and behavioural measures of the same construct.

\section{Conclusions}

Overall, the pattern of age effects in predictor, outcome, and cognitive control variables are broadly consistent with continuing maturation across adolescence into young adulthood, and plateau thereafter. Age effects were stronger on measures that tapped into cognitive control processes and controlled decisionmaking to optimise outcomes, consistent with adolescent risktaking models that argue for delayed maturation of cognitive control as compared to reward drive processes. Also, consistent with these models, self-reported level of cognitive control mediated the relationship between self-reported levels of impulsivity and psychological distress. However, first, this effect that was not moderated by age, suggesting that individual variability in cognitive control is more crucial to the relationship between risktaking/impulsivity and outcomes than age itself. Second, the mediation effect was significant with self-report but not behavioural or EEG-based measures of cognitive control. Finally, unlike impulsivity, self-reported level of perceived reward-seeking (benefit of risky behaviours, sensation-seeking) did not correlate with psychological distress or quality of life.

Acknowledgments This research was supported by an Australian Research Council Discovery Project (DP120100340 and DP170100756). PS and MM were supported by Australian Postgraduate Awards.

\section{References}

Allom, V., Panetta, G., Mullan, B., \& Hagger, M. S. (2016). Self-report and behavioural approaches to the measurement of self-control: Are we assessing the same construct?. Personality and Individual Differences, 90, 137-142.

Antony, M., Bieling, P., Cox, B., Enns, M., \& Swinson, R. (1998). Psychometric properties of the 42-item and 21-item versions of the Depression Anxiety Stress Scales in clinical groups and a community sample. Psychological Assessment, 10(2), 176-181.

Baron, R. M., \& Kenny, D. A. (1986). The Moderator-Mediator Variable Distinction in Social Psychological Research: Conceptual, Strategic, and Statistical Considerations. Journal of Personality and Social Psychology, 51(6), 1173-1182.

Benjamini, Y., \& Yekutieli, D. (2001). The control of the false discovery rate in multiple testing under dependency. Annals of statistics, 11651188.

Bernaards, C. A., \& Jennrich, R. I. (2005). Gradient projection algorithms and software for arbitrary rotation criteria in factor analysis. Educational and Psychological Measurement, 65(5), 676-696.

Bjork, J. M., \& Pardini, D. A. (2015). Who are those "risk-taking adolescents"? Individual differences in developmental neuroimaging research. Developmental cognitive neuroscience, 11, 56-64.

Blakemore, S. J., \& Choudhury, S. (2006). Development of the adolescent brain: implications for executive function and social cognition. Journal of child psychology and psychiatry, 47(3-4), 296-312.
Bø, R., Billieux, J., Gjerde, L. C., Eilertsen, E. M., \& Landrø, N. I. (2017). Do executive functions predict binge-drinking patterns? Evidence from a longitudinal study in young adulthood. Frontiers in psychology, 8, 489.

Bode, S., Feuerriegel, D., Bennett, D., \& Alday P. M. (2018). The Decision Decoding ToolBOX (DDTBOX) - A Multivariate Pattern Analysis Toolbox for Event-Related Potentials. Neuroinformatics. 1-16.

Braver, T. S. (2012). The variable nature of cognitive control: a dual mechanisms framework. Trends Cogn Sci, 16(2), 106-113.

Burgess, P., Alderman, N., Evans, J., Emslie, H., \& Wilson, B. (1998). The ecological validity of tests of executive function. Journal of the International Neuropsychological Society, 4(6), pp. 547-558.

Cambridge Cognition Ltd. CANTAB;( 2006). CANTABeclipse Test Administration Guide. Cambridge: Cambridge Cognition Ltd.

Casey, B. J. (2015). Beyond simple models of self-control to circuit-based accounts of adolescent behavior. Annual Review of Psychology, 66, 295-319.

Casey, B. J., Jones, R. M., \& Hare, T. A. (2008). The adolescent brain. Annals of the New York Academy of Sciences, 1124(1), 111-126.

Casey, B. J., Jones, R. M., \& Somerville, L. H. (2011). Braking and accelerating of the adolescent brain. Journal of Research on Adolescence, 21(1), 21-33.

Castellanos-Ryan, N., Parent, S., Vitaro, F., Tremblay, R. E., \& Séguin, J. R. (2013). Pubertal development, personality, and substance use: A 10 -year longitudinal study from childhood to adolescence. Journal of Abnormal Psychology, 122, 782-796. https://doi.org/10.1037/ a0033133

Catts, V. S., Fung, S. J., Long, L. E., Joshi, D., Vercammen, A., Allen, K. M., ... \& Tiwari, Y. (2013). Rethinking schizophrenia in the context of normal neurodevelopment. Frontiers in Cellular Neuroscience, 7, 60.

Coch, D., \& Gullick, M. M. (2012). Event-related potentials and development. The Oxford handbook of event-related potential components, 473-511.

Cooper, P. S., Wong, A. S., Fulham, W. R., Thienel, R., Mansfield, E., Michie, P. T., \& Karayanidis, F. (2015). Theta frontoparietal connectivity associated with proactive and reactive cognitive control processes. Neuroimage, 108, 354-363.

Cortes, C., \& Vapnik, V. (1995). Support-vector networks. Machine learning, 20(3), 273-297.

Cyders, M. A., \& Coskunpinar, A. (2011). Measurement of constructs using self-report and behavioral lab tasks: is there overlap in nomothetic span and construct representation for impulsivity?. Clinical psychology review, 31(6), 965-982.

Dawe, S., \& Loxton, N. J. (2004). The role of impulsivity in the development of substance use and eating disorders. Neuroscience \& Biobehavioral Reviews, 28(3), 343-351.

Derefinko, K. J., Peters, J. R., Eisenlohr-Moul, T. A., Walsh, E. C., Adams, Z. W., \& Lynam, D. R. (2014). Relations between trait impulsivity, behavioral impulsivity, physiological arousal, and risky sexual behavior among young men. Archives of Sexual Behavior, 43, 1149-1158. doi:https://doi.org/10.1007/s10508-014-0327-x

Donohew, L., Zimmerman, R., Cupp, P. S., Novak, S., Colon, S., \& Abell, R. (2000). Sensation seeking, impulsive decision-making, and risky sex: Implications for risk-taking and design of interventions. Personality and individual differences, 28(6), 1079-1091.

Duell, N., Steinberg, L., Chein, J., Al-Hassan, S. M., Bacchini, D., Lei, C., ... \& Lansford, J. E. (2016). Interaction of reward seeking and self-regulation in the prediction of risk taking: A cross-national test of the dual systems model. Developmental psychology, 52(10), 1593.

Eshel, N., Nelson, E. E., Blair, R. J., Pine, D. S., \& Ernst, M. (2007). Neural substrates of choice selection in adults and adolescents: development of the ventrolateral prefrontal and anterior cingulate cortices. Neuropsychologia, 45(6), 1270-1279. 
Fan, R. E., Chang, K. W., Hsieh, C. J., Wang, X. R., \& Lin, C. J. (2008). LIBLINEAR: A Library for Large Linear Classification. Journal of Machine Learning Research, 9, 1871-1874.

Fecteau, S., Knoch, D., Fregni, F., Sultani, N., Boggio, P., \& PascualLeone, A. (2007). Diminishing risk-taking behavior by modulating activity in the prefrontal cortex: a direct current stimulation study. Journal of Neuroscience, 27(46), 12500-12505.

Fromme, K., Katz, E., \& Rivet, K. (1997). Outcome expectancies and risktaking behavior. Cognitive Therapy And Research, 21(4), 421-442.

Gaston, K. J., Chown, S. L., \& Evans, K. L. (2008). Ecogeographical rules: elements of a synthesis. Journal of Biogeography, 35(3), 483-500.

Gogtay, N., Giedd, J. N., Lusk, L., Hayashi, K. M., Greenstein, D., Vaituzis, A. C., ... \& Rapoport, J. L. (2004). Dynamic mapping of human cortical development during childhood through early adulthood. Proceedings of the National academy of Sciences of the United States of America, 101(21), 8174-8179.

Gratton, C., Sun, H., \& Petersen, S. E. (2018). Control networks and hubs. Psychophysiology, 55(3), e13032.

Gratton, G., Cooper, P., Fabiani, M., Carter, C. S., \& Karayanidis, F. (2018). Dynamics of cognitive control: Theoretical bases, paradigms, and a view for the future. Psychophysiology, 55(3), e13016.

Gullo, M. J., \& Dawe, S. (2008). Impulsivity and adolescent substance use: Rashly dismissed as "all-bad"?. Neuroscience \& Biobehavioral Reviews, 32(8), 1507-1518.

Harden, K. P., Kretsch, N., Mann, F. D., Herzhoff, K., Tackett, J. L., Steinberg, L., \& Tucker-Drob, E. M. (2017). Beyond dual systems: A genetically-informed, latent factor model of behavioral and selfreport measures related to adolescent risk-taking. Developmental cognitive neuroscience, 25, 221-234.

Hayes, A. F. (2017). Introduction to mediation, moderation, and conditional process analysis: A regression-based approach. Guilford Publications.

Horn, J. L. (1965). A rationale and test for the number of factors in factor analysis. Psychometrika, 30(2), 179-185.

Hornik, K., Leisch, F., \& Zeileis, A. (2003). JAGS: A program for analysis of Bayesian graphical models using Gibbs sampling. In Proceedings of DSC (Vol. 2, pp. 1).

Hwang, K., Velanova, K., \& Luna, B. (2010). Strengthening of top-down frontal cognitive control networks underlying the development of inhibitory control: a functional magnetic resonance imaging effective connectivity study. Journal of Neuroscience, 30(46), 15535-15545.

Hyvärinen, A., \& Oja, E. (2000). Independent component analysis: algorithms and applications. Neural networks, 13(4-5), 411-430.

Imai, K., Keele, L., \& Tingley, D. (2010). A general approach to causal mediation analysis. Psychological methods, 15(4), 309.

Jamadar, S., Thienel, R., Karayanidis, F. (2015). Task switching. In: Toga AW (Ed). Brain mapping: An encyclopedic reference. (pp. 327-335) Amsterdam: Elsevier. ISBN: 978-0-12-397316-0

Jolliffe, I. (2011). Principal component analysis. In International encyclopedia of statistical science (pp. 1094-1096). Springer, Berlin, Heidelberg.

Karayanidis, F., Jamadar, S., \& Sanday, D. (2013). Stimulus-level interference disrupts repetition benefit during task switching in middle childhood. Frontiers in human neuroscience, 7, 841-

Karayanidis, F., Jamadar, S., Ruge, H., Phillips, N., Heathcote, A., \& Forstmann, B. U. (2010). Advance preparation in task-switching: converging evidence from behavioral, brain activation, and modelbased approaches. Frontiers in Psychology, 1, 25.

Karayanidis, F., Keuken, M. C., Wong, A., Rennie, J. L., de Hollander, G., Cooper, P. S., ... \& Michie, P. T. (2016). The Age-ility Project (Phase 1): Structural and functional imaging and electrophysiological data repository. Neuroimage, 124, 1137-1142.

Karayanidis, F., Mansfield, E. L., Galloway, K. L., Smith, J. L., Provost, A., \& Heathcote, A. (2009). Anticipatory reconfiguration elicited by fully and partially informative cues that validly predict a switch in task. Cognitive, Affective, \& Behavioral Neuroscience, 9(2), 202215.

Kass, R. E., \& Raftery, A. E. (1995). Bayes factors. Journal of the american statistical association, 90(430), 773-795.

Kayser, J., \& Tenke, C. E. (2006). Principal components analysis of Laplacian waveforms as a generic method for identifying ERP generator patterns: I. Evaluation with auditory oddball tasks. Clinical neurophysiology, 117(2), 348-368.

Kayser, J., \& Tenke, C. E. (2015). Issues and considerations for using the scalp surface Laplacian in EEG/ERP research: A tutorial review. International Journal of Psychophysiology, 97(3), 189-209.

Kessler, R., Andrews, G., Colpe, L., Hiripi, E., Mroczek, D., \& Normand, S. et al. (2002). Short screening scales to monitor population prevalences and trends in non-specific psychological distress. Psychological Medicine, 32(6), 959-976.

Koolschijn, P. C. M., Schel, M. A., de Rooij, M., Rombouts, S. A., \& Crone, E. A. (2011). A three-year longitudinal functional magnetic resonance imaging study of performance monitoring and test-retest reliability from childhood to early adulthood. Journal of Neuroscience, 31(11), 4204-4212.

Laurens, K. R., Hodgins, S., Maughan, B., Murray, R. M., Rutter, M. L., \& Taylor, E. A. (2007). Community screening for psychotic-like experiences and other putative antecedents of schizophrenia in children aged 9-12 years. Schizophrenia research, 90(1), 130-146.

Lezak, M., Howieson, D., Loring, D., \& Fischer, J. (2004). Neuropsychological assessment (4th ed.). Oxford: Oxford University Press.

Liang, F., Paulo, R., Molina, G., Clyde, M. A., \& Berger, J. O. (2008). Mixtures of g priors for Bayesian variable selection. Journal of the American Statistical Association, 103(481), 410-423.

Los, S. A. (1996). On the origin of mixing costs: Exploring information processing in pure and mixed blocks of trials. Acta Psychologica, 94(2), 145-188.

Luna, B., Garver, K. E., Urban, T. A., Lazar, N. A., \& Sweeney, J. A. (2004). Maturation of cognitive processes from late childhood to adulthood. Child development, 75(5), 1357-1372.

MacKillop, J., Weafer, J., Gray, J. C., Oshri, A., Palmer, A., \& de Wit, H. (2016). The latent structure of impulsivity: impulsive choice, impulsive action, and impulsive personality traits. Psychopharmacology, 233(18), 3361-3370.

MacKinnon, D. (2008). Introduction to statistical mediation analysis. Routledge, New York.

Magar, E. C., Phillips, L. H., \& Hosie, J. A. (2008). Self-regulation and risk-taking. Personality and individual differences, 45(2), 153-159.

McAuley, T., Chen, S., Goos, L., Schachar, R., \& Crosbie, J. (2010). Is the Behavior Rating Inventory of Executive Function more strongly associated with measures of impairment or executive function? Journal of the International Neuropsychological Society, 16(3), 495-505.

Moffitt, T. E., Arseneault, L., Belsky, D., Dickson, N., Hancox, R. J., Harrington, H., ... \& Sears, M. R. (2011). A gradient of childhood self-control predicts health, wealth, and public safety. Proceedings of the National Academy of Sciences, 108(7), 2693-2698.

Munakata, Y., Snyder, H. R., \& Chatham, C. H. (2012). Developing cognitive control: Three key transitions. Current directions in psychological science, 21(2), 71-77.

Nigg, J. T. (2017). Annual Research Review: On the relations among selfregulation, self-control, executive functioning, effortful control, cognitive control, impulsivity, risk-taking, and inhibition for developmental psychopathology. Journal of child psychology and psychiatry, 58(4), 361-383.

Nuijten, M. B., Wetzels, R., Matzke, D., Dolan, C. V., \& Wagenmakers, E. J. (2015). A default Bayesian hypothesis test for mediation. Behavior research methods, 47(1), 85-97. doi: https://doi.org/10. 3758/s13428-014-0470-2. 
Oostenveld, R., Fries, P., Maris, E., \& Schoffelen, J. M. (2011). FieldTrip: open source software for advanced analysis of MEG, EEG, and invasive electrophysiological data. Computational intelligence and neuroscience, 2011, 1 .

Osborne, J. W., \& Costello, A. B. (2004). Sample size and subject to item ratio in principal components analysis. Practical assessment, research \& evaluation, $9(11), 8$.

Osman, A., Bagge, C., Gutierrez, P., Konick, L., Kopper, B., \& Barrios, F. (2001). The Suicidal Behaviors Questionnaire-Revised (SBQ-R): Validation with Clinical and Nonclinical Samples. Assessment, 8(4), 443-454.

Patton, J., Stanford, M., \& Barratt, E. (1995). Factor structure of the barratt impulsiveness scale. Journal Of Clinical Psychology, 51(6), 768-774.

Paus, T. (2005). Mapping brain maturation and cognitive development during adolescence. Trends in cognitive sciences, 9(2), 60-68.

Peeters, M., Oldehinkel, T., \& Vollebergh, W. (2017). Behavioral control and reward sensitivity in adolescents' risk taking behavior: A longitudinal TRAILS study. Frontiers in psychology, 8, 231.

Pfeifer, J. H., \& Allen, N. B. (2012). Arrested development? Reconsidering dual-systems models of brain function in adolescence and disorders. Trends in cognitive sciences, 16(6), 322-329.

R Core Team (2017). R: A Language and Environment for Statistical Computing. Retrieved from https://www.R-project.org/

Rabin, L. A., Roth, R. M., Isquith, P. K., Wishart, H. A., Nutter-Upham, K. E., Pare, N., ... \& Saykin, A. J. (2006). Self-and informant reports of executive function on the BRIEF-A in MCI and older adults with cognitive complaints. Archives of Clinical Neuropsychology, 21(7), 721-732.

Reitan, R. M. (1959). The comparative effects of brain damage on the Halstead Impairment Index and the Wechsler-Bellevue Scale. Journal of clinical psychology, 15(3), 281-285.

Reuter-Lorenz, P. A., \& Cappell, K. A. (2008). Neurocognitive aging and the compensation hypothesis. Current directions in psychological science, 17(3), 177-182.

Revelle, W. (2018). psych: Procedures for Psychological, Psychometric, and Personality Research. R package version 1.8.3. Retrieved from, https://CRAN.R-project.org/package=psych

Reyna, V. F., \& Farley, F. (2006). Risk and rationality in adolescent decision making: Implications for theory, practice, and public policy. Psychological science in the public interest, 7(1), 1-44.

Ridderinkhof, K. R., Band, G. P., \& Logan, G. D. (1999). A study of adaptive behavior: Effects of age and irrelevant information on the ability to inhibit one's actions. Acta psychologica, 101(2-3), 315337.

Romer, D., Reyna, V. F., \& Satterthwaite, T. D. (2017). Beyond stereotypes of adolescent risk taking: Placing the adolescent brain in developmental context. Developmental cognitive neuroscience, 27, 19-34.

Roth, R. M., Isquith, P. K., \& Gioia, G. A. (2005). Behavior Rating Inventory of Executive Function - Adult Version. Psychological Assessment Resources.

Sander, M. C., Lindenberger, U., \& Werkle-Bergner, M. (2012). Lifespan age differences in working memory: A two-component framework. Neuroscience \& Biobehavioral Reviews, 36(9), 2007-2033.

Saunders, J. A., Morrow-Howell, N., Spitznagel, E., Doré, P., Proctor, E. K., \& Pescarino, R. (2006). Imputing missing data: A comparison of methods for social work researchers. Social work research, 30(1), 19-31.

Segalowitz, S. J., Santesso, D. L., \& Jetha, M. K. (2010). Electrophysiological changes during adolescence: a review. Brain and cognition, 72(1), 86-100.

Sharma, L., Markon, K. E., \& Clark, L. A. (2014). Toward a theory of distinct types of "impulsive" behaviors: A meta-analysis of self-report and behavioral measures. Psychological bulletin, 140(2), 374

Shulman, E. P., Smith, A. R., Silva, K., Icenogle, G., Duell, N., Chein, J., \& Steinberg, L. (2016). The dual systems model: Review, reappraisal, and reaffirmation. Developmental Cognitive Neuroscience, 17, 103-117.

Skippen, P., Matzke, D., Heathcote, A., Fulham, W. R., Michie, P., \& Karayanidis, F. (2019). Reliability of triggering inhibitory process is a better predictor of impulsivity than SSRT. Acta psychologica, 192, 104-117.

Somerville, L. H., Hare, T., \& Casey, B. J. (2011). Frontostriatal maturation predicts cognitive control failure to appetitive cues in adolescents. Journal of cognitive neuroscience, 23(9), 2123-2134.

Stahl, C., Voss, A., Schmitz, F., Nuszbaum, M., Tüscher, O., Lieb, K., \& Klauer, K. C. (2014). Behavioral components of impulsivity. Journal of Experimental Psychology: General, 143(2), 850.

Stanford, M. S., Greve, K. W., Boudreaux, J. K., Mathias, C. W., \& Brumbelow, J. L. (1996). Impulsiveness and risk-taking behavior: Comparison of high-school and college students using the Barratt Impulsiveness Scale. Personality and individual differences, 21(6), 1073-1075.)

Steinberg, L. (2007). Risk taking in adolescence: New perspectives from brain and behavioral science. Current Directions in Psychological Science, 16(2), 55-59.

Steinberg, L. (2009). Should the science of adolescent brain development inform public policy?. American Psychologist, 64(8), 739.

Steinberg, L., Albert, D., Cauffman, E., Banich, M., Graham, S., \& Woolard, J. (2008). Age differences in sensation seeking and impulsivity as indexed by behavior and self-report: evidence for a dual systems model. Developmental Psychology, 44(6), 1764.

Stevens, M. C., Kiehl, K. A., Pearlson, G. D., \& Calhoun, V. D. (2007). Functional neural networks underlying response inhibition in adolescents and adults. Behavioural brain research, 181(1), 12-22.

The WHOQOL Group. (1998). Development of the World Health Organization WHOQOL-BREF Quality of Life Assessment. Psychological Medicine, 28(3), 551-558. doi: https://doi.org/10. 1017/s0033291798006667

Tingley, D., Yamamoto, T., Hirose, K., Keele, L., \& Imai, K. (2014). Mediation: R package for causal mediation analysis. Journal of Statistical Software, 59(5), 1-38. doi: https://doi.org/10.18637/jss. v059.i05

Uhlhaas, P. J., Roux, F., Rodriguez, E., Rotarska-Jagiela, A., \& Singer, W. (2010). Neural synchrony and the development of cortical networks. Trends in cognitive sciences, 14(2), 72-80.

Van Leijenhorst, L., Moor, B. G., de Macks, Z. A. O., Rombouts, S. A., Westenberg, P. M., \& Crone, E. A. (2010). Adolescent risky decision-making: neurocognitive development of reward and control regions. Neuroimage, 51(1), 345-355.

Van Leijenhorst, L., Zanolie, K., Van Meel, C. S., Westenberg, P. M., Rombouts, S. A., \& Crone, E. A. (2009). What motivates the adolescent? Brain regions mediating reward sensitivity across adolescence. Cerebral cortex, 20(1), 61-69.

Wechsler, D. (2008). WAIS-IV administration and scoring manual. San Antonio, TX: Pearson.

Wechsler, D. (2011). Wechsler Abbreviated Scale of Intelligence-Second Edition (WASI-II). San Antonio, TX: Pearson

Zuckerman, M., Kolin, E., Price, L., \& Zoob, I. (1964). Development of a sensation-seeking scale. Journal of Consulting Psychology, 28(6), 477-482.

Publisher's note Springer Nature remains neutral with regard to jurisdictional claims in published maps and institutional affiliations. 